\title{
Brain- and brain tumor-penetrating disulfiram nanoparticles: Sequence of cytotoxic events and efficacy in human glioma cell lines and intracranial xenografts
}

\author{
Hanumantha Rao Madala ${ }^{1}$, Surendra R. Punganuru ${ }^{1}$, Francis Ali-Osman ${ }^{2}$, Ruiwen \\ Zhang $^{3}$ and Kalkunte S. Srivenugopal ${ }^{1}$ \\ ${ }^{1}$ Department of Biomedical Sciences, School of Pharmacy, Texas Tech University Health Sciences Center, Amarillo, TX, USA \\ ${ }^{2}$ Department of Surgery and the Brain Tumor Center, Duke University, Durham, NC, USA \\ ${ }^{3}$ Department of Pharmacological and Pharmaceutical Sciences, College of Pharmacy, University of Houston, Houston, TX, USA \\ Correspondence to: Kalkunte S. Srivenugopal, email: Kalkunte.Srivenugopal@ttuhsc.edu
}

Keywords: disulfiram; MGMT; glioma; chemotherapy; nanoparticles

Received: September 12,2017 Accepted: November 26, $2017 \quad$ Published: December 15, 2017

Copyright: Madala et al. This is an open-access article distributed under the terms of the Creative Commons Attribution License 3.0 (CC BY 3.0), which permits unrestricted use, distribution, and reproduction in any medium, provided the original author and source are credited.

\section{ABSTRACT}

There is great interest in repurposing disulfiram (DSF), a rapidly metabolizing nontoxic drug, for brain cancers and other cancers. To overcome the instability and low therapeutic efficacy, we engineered passively-targeted DSF-nanoparticles (DSFNPs) using biodegradable monomethoxy (polyethylene glycol) d,l-lactic-co-glycolic acid (mPEG-PLGA) matrix. The physicochemical properties, cellular uptake and the blood brain-barrier permeability of DSFNPs were investigated. The DSFNPs were highly stable with a size of $\sim 70 \mathrm{~nm}$ with a $>\mathbf{9 0 \%}$ entrapment. Injection of the nanoparticles labeled with HITC, a near-infrared dye into normal mice and tumor-bearing nude mice followed by in vivo imaging showed a selective accumulation of the formulation within the brain and subcutaneous tumors for $>24 \mathrm{~h}$, indicating an increased plasma halflife and entry of DSF into desired sites. The DSFNPs induced a potent and preferential killing of many brain tumor cell lines in cytotoxicity assays. Confocal microscopy showed a quick internalization of the nanoparticles in tumor cells followed by initial accumulation in lysosomes and subsequently in mitochondria. DSFNPs induced high levels of ROS and led to a marked loss of mitochondrial membrane potential. Activation of the MAP-kinase pathway leading to a nuclear translocation of apoptosisinducing factor and altered expression of apoptotic and anti-apoptotic proteins were also observed. DSFNPs induced a powerful and significant regression of intracranial medulloblastoma xenografts compared to the marginal efficacy of unencapsulated DSF. Together, we show that passively targeted DSFNPs can affect multiple targets, trigger potent anticancer effects, and can offer a sustained drug supply for brain cancer treatment through an enhanced permeability retention (EPR).

\section{INTRODUCTION}

Disulfiram (DSF, Antabuse) was the first drug approved by the U.S. Food and Drug Administration to treat chronic alcohol dependence [1]. DSF (tetraethylthiuram disulfiram) is a hydrophobic, symmetrical molecule of the dithiocarbamate family and is available in oral tablets. The drug has a strong affinity for protein-bound and unbound thiols and forms a covalent linkage with an active-site cysteine of the aldehyde dehydrogenase (ALDH) to inactivate the enzyme and build up the acetaldehyde concentrations to cause aversion to alcohol. Recently, DSF has gained prominence as a potent anticancer drug, owing to its pharmacological actions on multiple targets in tumor cells. It has been shown to trigger oxidative stress by generating reactive oxygen species (ROS) [2], abrogate the superoxide dismutase (SOD) activity [3] and activate the mitogen-activated protein kinase (MAPK) pathway 
[4]. DSF can reverse the resistance to chemotherapy drugs by inhibiting the P-glycoprotein (Pgp) multidrug efflux pump [5]. It was shown to inhibit the activation of nuclear factor-kB (NF-kB) a well-known resistance marker $[4,6]$. In addition, DSF has been found to reduce angiogenesis because of its metal chelating properties, inactivation of $\mathrm{Cu} / \mathrm{Zn}$ superoxide dismutase (SOD) and matrix metalloproteinases $[3,7]$. DSF has also been shown to inhibit the proteasome [5], DNA topoisomerases [8], DNA methyltransferase (DNMT) [9] and glutathione S-transferase P1 (unpublished). More importantly, related to this study, our laboratory has demonstrated the ability of DSF and to inhibit $\mathrm{O}^{6}$-methylguanine DNA methyltransferase (MGMT), a DNA repair protein highly expressed in brain tumors [10]. MGMT normally functions to protect the genome against mutations generated by the pairing of $\mathrm{O}^{6}$-alkylguanines with thymine. Paradoxically, the higher MGMT content in gliomas serves to remove the mutagenic and cytotoxic $\mathrm{O}^{6}$-alkylguanines and confer tumor drug resistance and therapy failure [11]. Consistent with this observation, we have also found that different dithiocarbamates also retained the ability to inactivate MGMT [12]. Because DSF bears several properties, including the ability to cross the blood-brain barrier (BBB) [13], cytotoxicity against glioma stem cells [14], inhibition of various targets, including the MGMT and ALDH [15], there is significant interest in developing DSF for brain tumor treatment; in fact, a clinical trial has already been listed [16]. DSF with or without soluble copper (Cugluconate) is also undergoing clinical trials in liver cancer and other cancer types $[17,18]$.

After absorption, disulfiram is rapidly split into its half-molecule diethyldithiocarbamate (DDC), which readily complexes with copper in the stomach. Consequently, absorption and distribution via the gastrointestinal mucosa to the blood might involve both the parent drug and its copper diethyldithiocarbamic acid (DDC), which is unstable and is further degraded to form diethylamine and carbon disulfide [19]. DDC is also a substrate for phase II metabolism, which involves the formation of diethyldithiomethylcarbamate (Me-DDC) and the glucuronic acid of DDC. Me-DDC also undergoes oxidative biotransformation to diethylthiomethylcarbamate (Me-DTC) and is further oxidized to its corresponding sulfoxide and sulfone metabolites. Surprisingly, it was found that the plasma concentration of DSF after oral administration of a 500-mg tablet was below the limit of detection which explains the limitations of the DSF as a drug in a clinical setting [19-21]. Its extreme instability in the gastric environment and rapid degradation by the glutathione reductase in plasma appear to cause an unfavorable pharmacokinetics, which is hampering its further development as an anticancer drug [19].

The development of drug carriers, which deliver the drugs selectively to the site of action with a controlled rate of release has been a goal of drug therapy. Among various types of drug carriers, polymeric nanoparticles are advantageous in terms of stability, flexibility to control the rate of drug release and stealth behavior in circulation. Poly(Lactide-co-Glycolide) or PLGA is an FDA approved biodegradable polymer that is physically strong, highly biocompatible and with an established use for delivering drugs, proteins, and nucleic acids. A long clinical experience, favorable degradation characteristics and possibilities for sustained drug delivery are other advantages of PLGA. PLGA undergoes hydrolysis to yield the monomers (i.e., lactic and glycolic acid) which are endogenous and are metabolized by the body via Krebs cycle resulting in minimal systemic toxicity [22]. Polyethylene glycol (PEG) forms a hydrophilic coat over the hydrophobic PLGA core and protects the formulation from being engulfed by the reticuloendothelial cells (RECs) giving a stealth behavior and confers long circulation properties by acting as steric stabilizer [23]. PEG minimizes the ionic strength which stabilizes the nanoparticles from aggregation in the physiological solutions [23]. Polymeric nanoparticles with particle sizes within the range of $100 \mathrm{~nm}$ or less can take advantage of the enhanced permeability and retention (EPR) effect, which exists in areas of new blood vessel growth. For example, the EPR effect can be used to deliver anti-cancer drugs into tumor tissues via passive targeting [24]. EPR is also known to impart less toxicity because of the preferential accumulation of the passive formulations at tumor sites and limit off-target side effects [24-26]. Enhanced EPR effects prevalent in disrupted blood brain-barrier found in CNS cancers may further aid a greater entry of nanoparticles.

With the above considerations in mind, this study applied the most common and well-known mPEG: PLGA polymer for DSF encapsulation. PLGA forms a hydrophobic core in which the DSF gets encapsulated, and the surface of the core is covered with hydrophilic PEG chains (Figure 1A). There are a few reports of DSF nanoformulations with active targeting such as folate receptors targeted nanoparticles for breast cancer [25], lipid nanocapsules modified with cell penetrating peptide (TAT) for hepatic cancer [26], mPEG-PLGA/PCL nanoparticles for breast cancer [20], hot melt extruded and injection-molded PLGA millirods for GBM [27]. However, none of these have entered clinical trials; therefore, we considered a simpler approach to encapsulate DSF in mPEG: PLGA nanoparticles (DSFNPs). It is noteworthy that most of the FDA approved nanoformulations are made with PEG and all of them are passively targeted, despite the known advantages of specific delivery through activetargeting strategies $[27,28]$. The current study prepared and optimized a formulation of DSF and characterized its physicochemical properties. The preferential accumulation of DSFNPs in the brain and the cellular pathways leading to the cytotoxicity were investigated. We also show DSFNPs exert strong antitumor effects in medulloblastoma orthotopic xenograft models. 


\section{RESULTS AND DISCUSSION}

\section{Preparation of DSFNPs and optimization of formulation}

We used the single emulsion solvent evaporation technique, a proven and successful procedure for preparing DSF nanoparticles of hydrophobic drugs as described in Methods. The process and formulation variables play a key role in acquiring homogenous particles with a lower size and higher encapsulation efficiency. We found nanoparticles that were prepared using watermiscible solvents had better features compared to the water immiscible or partially miscible solvents. A ratio of 1:10 of organic/aqueous phase and drug/polymer resulted in optimal size and encapsulation. The presence or absence of surfactants with water-miscible solvents did not significantly influence on size or encapsulation.

A

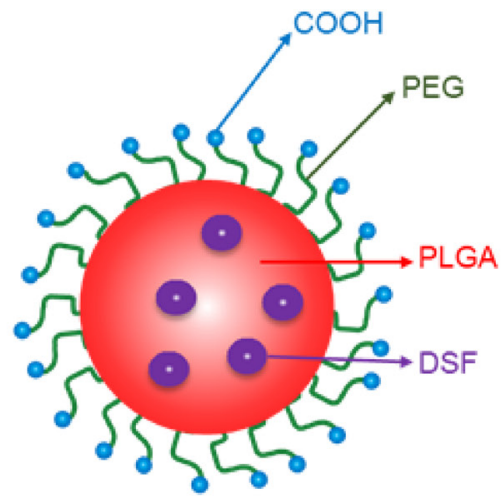

B

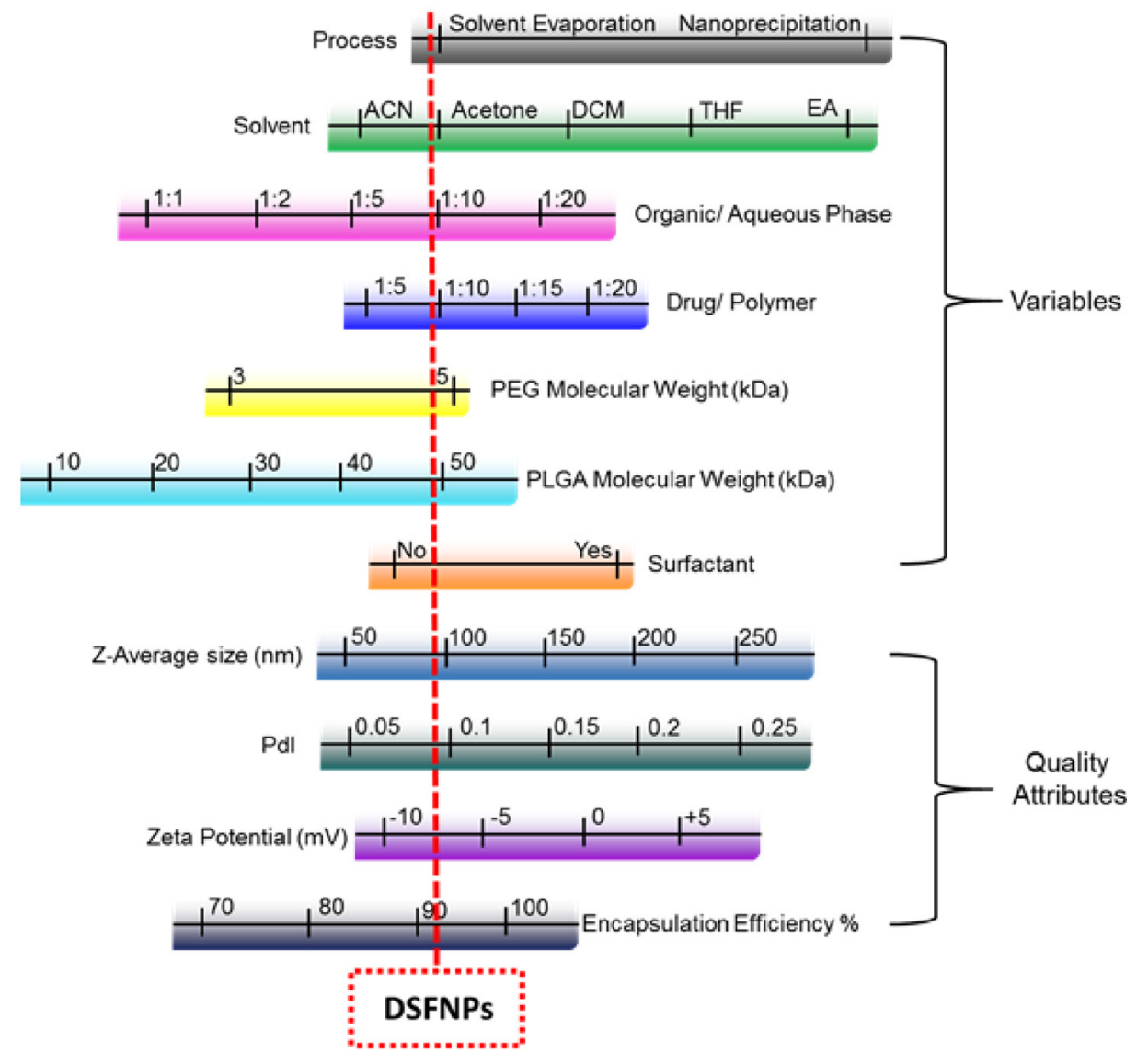

Figure 1: Representation of a disulfiram encapsulated nanoparticle and summary of optimization of DFSNPs. (A) Schematic representation of a typical nanoparticle. (B). Ranges of formulation parameters and NP physicochemical properties evaluated during the development of DSFNPs. The red dotted line indicates the parameters of the finally developed DSFNPs, which was considered optimal for experimental and therapeutic purposes. 
Among the various sizes of mPEG: PLGA polymers tested, the mPEG $(5 \mathrm{kDa})$ : PLGA $(45 \mathrm{kDa})$ resulted in an optimal size and greater encapsulation efficiency. Figure 1B summarizes the formulation variables we tested in optimizing the physicochemical parameters of the DSFNPs. The procedural details and results of the DSFNPs optimization are provided in supplementary data (Supplementary Figures 1-3, Supplementary Table 1). Altogether, the formulation 1, prepared by solvent evaporation using acetone, mPEG (5 kDa): PLGA (45 kDa) polymer, without surfactant and at organic/aqueous phase and drug/polymer ratio of 1:10 gave the best size $(\sim 70-80 \mathrm{~nm})$, PDI $(<0.2)$ and $\%$ EE of $>90 \%$. We used this formulation 1 for further characterization.

Both the blank and DSF encapsulated nanoparticles (DSFNPs) were analyzed for the size and the size distribution by dynamic light scattering (DLS) and representative size distribution plots are shown in Figure $2 \mathrm{~A}, 2 \mathrm{~B}$. The monomodal distribution of the formulation was further confirmed with transition electron microscopy (TEM) (Figure 2C, 2D). Both DLS and TEM determined the average diameter of the blank NPs and DSFNPs in the range of 72-76 nm with a PDI of 0.16 and 0.18 respectively. The scanning electron microscopy (SEM) confirmed the spherical shape of the DSFNPs (Figure 2E, $2 F)$. The zeta potentials of the blank NPs and DSFNPs were found to be -6.26 to $-8.27 \mathrm{mV}$. The HITC- nearinfrared dye incorporated nanoparticles (HITCNPs) were also found to have similar parameters as that of DSFNPs (Data not shown). The drug loading and encapsulation efficiency were determined to be $18.47 \%$ and $92.1 \%$ as summarized in Figure 2G.

\section{X-Ray diffraction analysis}

XRD patterns of DSF, mPEG: PLGA polymer and DSFNPs are shown in Figure 3A. Characteristic diffraction peaks were observed for DSF free drug, whereas, no such intense diffraction peaks were apparent with the DSFNPs and the overall curve shape was similar to mPEG: PLGA. These results indicated complete encapsulation of DSF in the polymer matrix with the reduction in the crystallinity of the DSFNPs compared to the free drug.

\section{Differential scanning calorimetry}

The thermograms define the physical states of the drug and the polymer and are useful in detecting any drug-polymer interactions within the polymeric network of the NPs. Differential scanning calorimetry (DSC) thermograms of DSF, mPEG: PLGA and DSFNPs are presented in Figure 3B. The DSF thermogram displayed an endothermic peak at $71.659^{\circ} \mathrm{C}$ and mPEG: PLGA did not show any endothermic peak in the temperature range tested, indicating the amorphous nature of the polymer.
Whereas, DSFNPs showed a small endothermic peak at $70.911^{\circ} \mathrm{C}$. These results indicated that there was no crystalline drug material on the surface of the formulation and confirmed its complete encapsulation. Further, it can be concluded that disulfiram in DSFNPs was present in the amorphous phase in a homogeneous dispersion within the PLGA matrix.

\section{Thermogravimetric analysis (TGA)}

TGA analysis gave the degradation points of DSF, mPEG: PLGA and DSFNPs as shown in Figure 3C. The first depression in the curves was a measure of moisture content and we did not see any depression in DSFNPs until $300^{\circ} \mathrm{C}$ indicating no moisture in DSFNPs. DSF started losing the weight from $193^{\circ} \mathrm{C}(95 \%)$ and it reached $5 \%$ at $279^{\circ} \mathrm{C}$. Polymer, mPEG: PLGA lost $5 \%$ of weight at $281{ }^{\circ} \mathrm{C}$ and at $400^{\circ} \mathrm{C}$ it lost $95 \%$ of its weight. Whereas, the DSFNPs lost their weight by $5 \%$ at $217^{\circ} \mathrm{C}$ and $36 \%$ at $800^{\circ} \mathrm{C}$ leaving almost $64 \%$ of residue at the end.

\section{In vitro drug-release kinetics}

In vitro drug release kinetics provide critical information about dosage form behavior and is a key parameter used to assess product safety and efficacy. The stability and controlled drug release profile of DSFNPs was assessed by measuring the cumulative release of DSF at physiological $\mathrm{pH}$ condition ( $\mathrm{pH}$ 7.4), in simulated intestinal fluid $(\mathrm{pH} 6.8)$, and in simulated gastric fluid (pH 1.2). $14.5 \%, 19.4 \%$, and $35.2 \%$ of the total DSF was released from the nanoparticles after $8 \mathrm{~h}$ at $\mathrm{pH} 7.4$, 6.8 , and 1.2 respectively, confirming that the majority of DSFNPs are stable under these conditions (Figure 3D). The cumulative drug release after $24 \mathrm{~h}$ was found to be $14.5 \%, 19.4 \%$, and $35.2 \%$, respectively, at above $\mathrm{pH}$ values and there was a steady release of DSF over the next nine days. These results provide strong evidence that DSFNPs are stable enough to allow a sustained release of the compound in both the stomach and intestine by protecting DSF from premature degradation. To gain a better understanding of the release profile of DSFNPs, we plotted the data as follows: cumulative $\%$ drug release vs time (Zero order kinetics model), log cumulative $\%$ drug remaining vs time (First-order kinetic model), cumulative $\%$ drug release vs square root of time (Higuchi model) and $\log$ cumulative $\%$ release vs log time (Korsmeyer- Peppas model). The $\mathrm{R}^{2}$ is a coefficient of determination, $\mathrm{k}$ is rate constant and $n$ is release exponent. On the basis of best fit with the highest $\mathrm{R}^{2}$ value, it is concluded that in the optimized formulation of nanoparticles follow the Higuchi model (data is not shown), which has a large application in polymeric matrix systems [30]. The Korsmeyer- Peppas model revealed the release mechanism follows Fickian diffusion with release exponent value $n \cong 0.5$ with all three buffers we tested. 

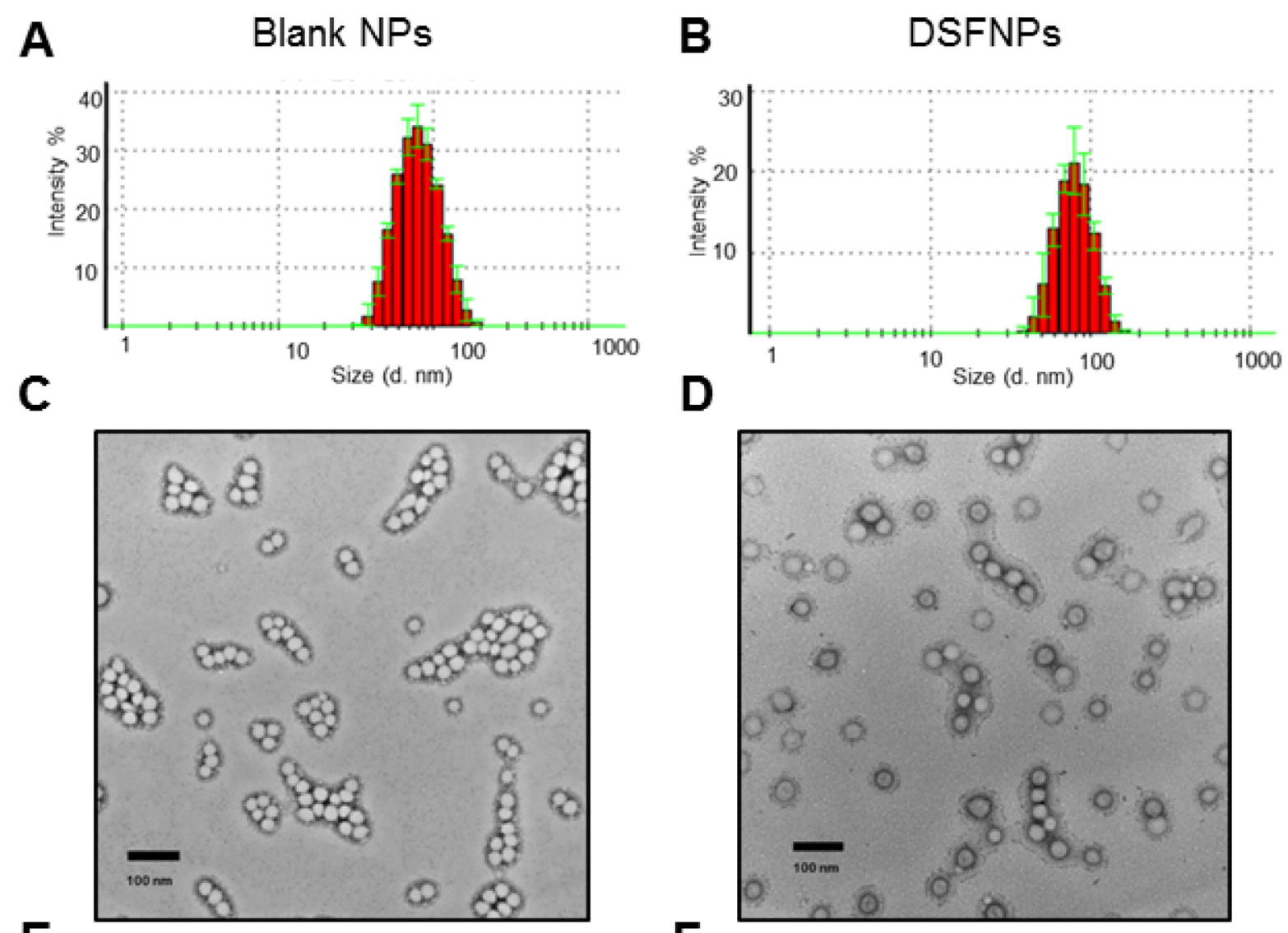

E

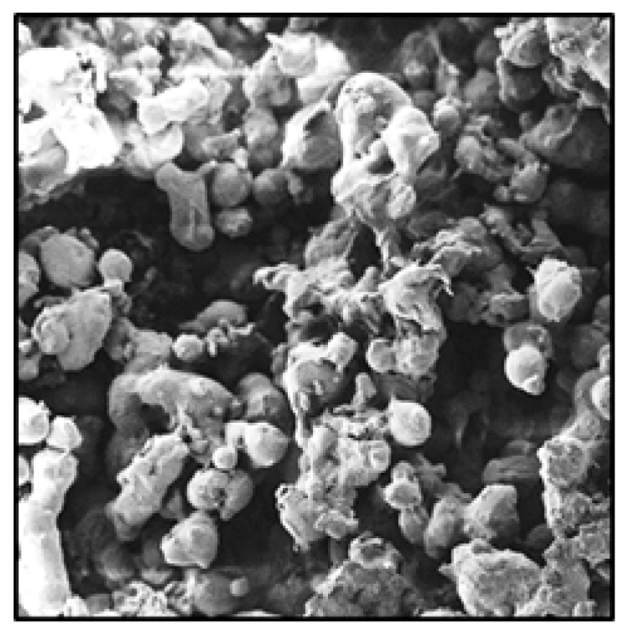

$G$

\begin{tabular}{cccccc} 
Formulation & $\begin{array}{c}\text { Particle Diameter } \\
(\mathrm{nm})\end{array}$ & Pdl & $\begin{array}{c}\text { Zeta Potential } \\
(\mathrm{mV})\end{array}$ & $\begin{array}{c}\text { Drug Loading } \\
(\%)\end{array}$ & $\begin{array}{c}\text { Encapsulation } \\
\text { Efficiency } \\
(\%)\end{array}$ \\
\hline Blank NPs & $76.34 \pm 3.87$ & $0.16 \pm 0.05$ & -6.26 & - & - \\
DSFNPs & $81.74 \pm 3.3$ & $0.18 \pm 0.06$ & -8.27 & 18.47 & 92.1
\end{tabular}

Figure 2: Characterization of mPEG-PLGA nanoparticles. (A, B) The size, size distribution and surface morphology of the blank NPs and DSFNPs as determined by DLS (C, D) TEM and (E, F) SEM. (G) The size (d. nm), zeta potential, PDI and \% EE of blank and DSFNPs. Data represent mean \pm SD. Experiments were performed in triplicate $(n=3)$. 


\section{In vitro permeability and cellular uptake of DSFNPs}

The effect of the encapsulation of DSF into mPEGPLGA nanoparticles on the permeability of the drug was investigated using IMR-90 cells, a well-characterized in vitro model for BBB permeability studies [29, 31]. As shown in Figure 4A-4B, the transepithelial transport of DSF was significantly enhanced by the nano-delivery system, in a time and dose-dependent manner. There was a five-fold increase in DSF transport from the nanoparticle groups when compared to DSF alone in IMR-90 cells after $2 \mathrm{~h}$ incubation. The apparent permeability coefficients $\left(P_{\text {app }}\right)$ of DSF and DSFNPs were $6.037 \times 10^{-8} \mathrm{~cm} / \mathrm{s}$ and $4.01 \times 10^{-7} \mathrm{~cm} / \mathrm{s}$ respectively. The transepithelial electrical resistance (TEER) was not affected by DSF and DSFNPs throughout the experiment, suggesting that the increased DSF transport was not due to a decrease in the monolayer integrity or the opening of tight junctions.

\section{In vitro uptake of DSFNPs by cancer cells}

Uptake of DSF and DSFNPs was investigated in the T98G and DAOY brain cancer cell lines. As shown in Figure 4D, 4G, the cellular uptake increased in a timedependent manner for both DSF and DSFNPs. Compared to the free DSF, there was 2-3 -fold higher cellular uptake of DSFNPs in both cell lines after a $2 \mathrm{~h}$ incubation. We observed a similar trend in the experiments performed with different doses of DSF and DSFNPs (Figure 4C, 4F).

Further, the nanoparticle-induced enhancement of the cancer cell uptake of the encapsulated drug was confirmed using HITC encapsulated NPs (HITCNPs). The fluorescence of intracellular HITC was determined by flow cytometry. The intensity and shifting of the fluorescence peaks shown in Figures 4E, 4H indicate that there was a significant increase in the uptake of disulfiram after formulation when compared to HITC alone in both the cell lines.

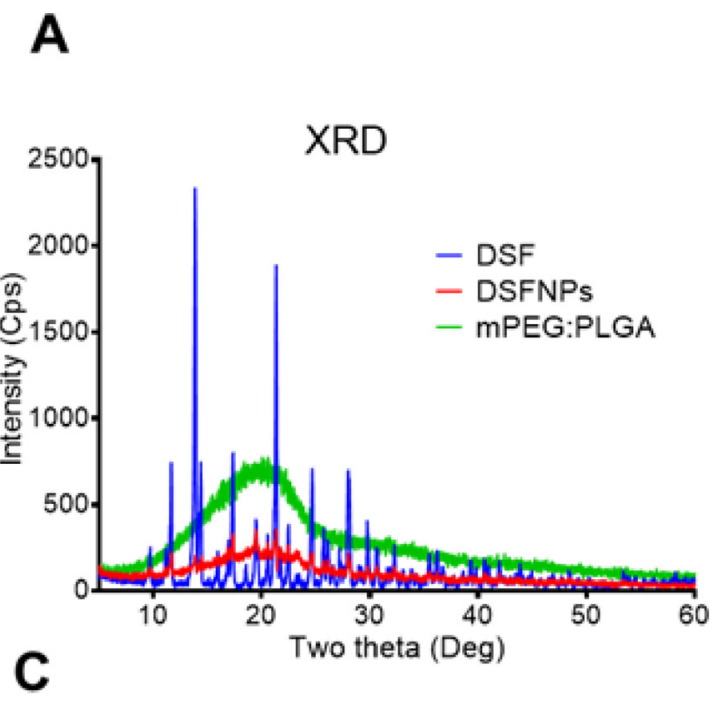

B
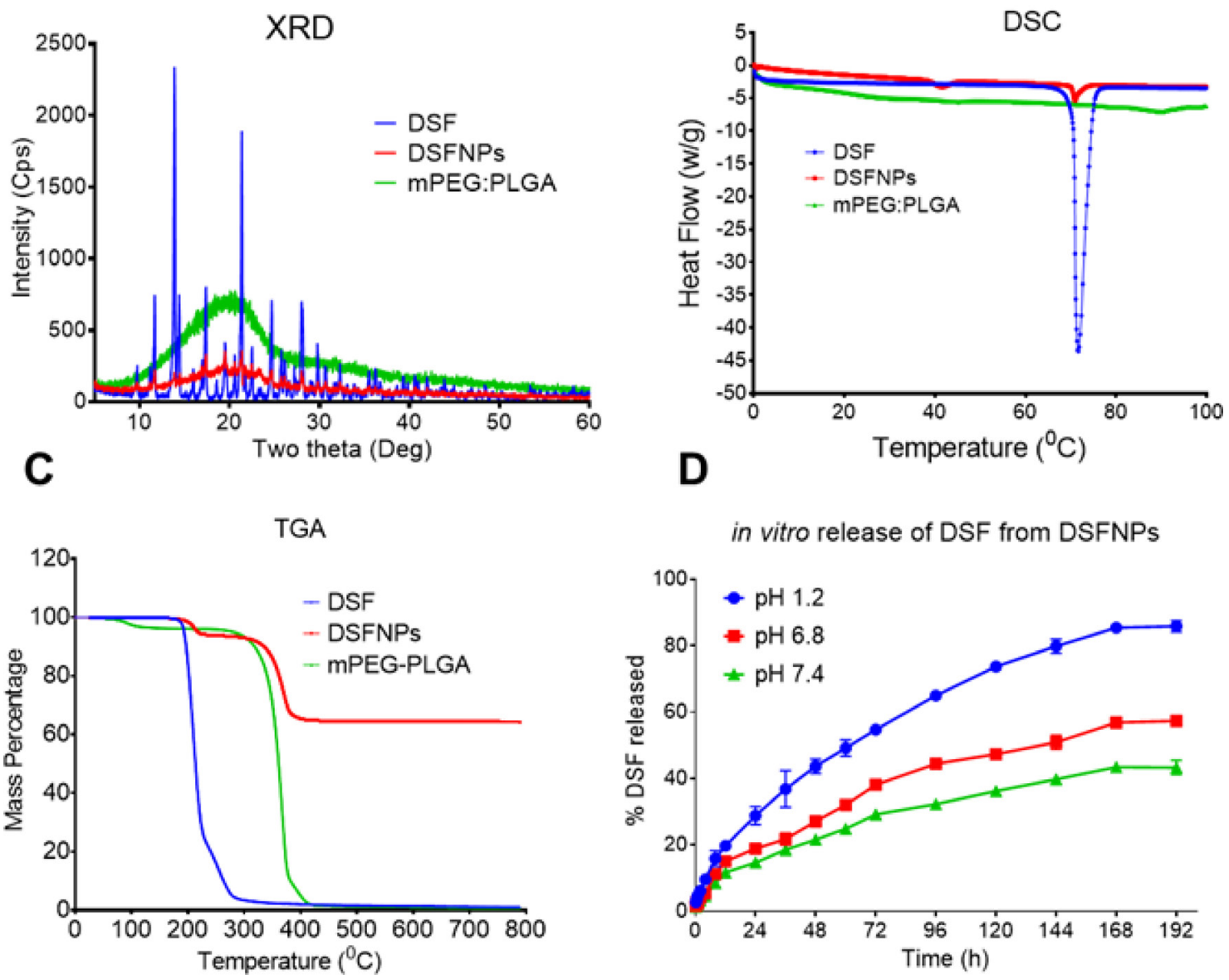

in vitro release of DSF from DSFNPs

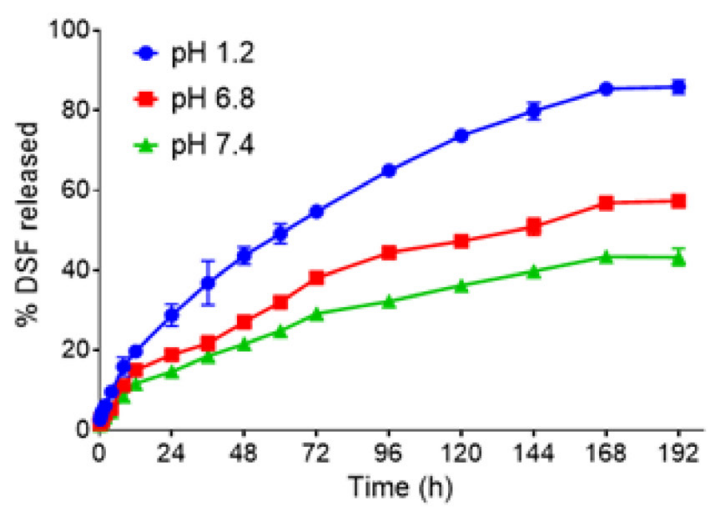

Figure 3: Physical characterization of DSFNPs. Results from the (A) XRD; (B) DSC and (C) TGA of DSF, DSFNPs and mPEG: PLGA are represented. (D). The cumulative release kinetics of DSF from DSFNP in simulated gastric fluid (pH 1.2), simulated intestinal fluid (pH 6.8) and PBS ( $\mathrm{pH}$ 7.4) are shown. The concentration units for DSFNPs and DSF used were equivalent in all experiments. Data represent mean $\pm \mathrm{SD}$. Experiments were performed in triplicate $(n=3)$. 


\section{Real-time imaging of near-infrared (NIR) dye HITC $\left(1,1^{\prime}, 3,3,3^{\prime}, 3^{\prime}\right.$-hexamethylindotrycarbo cyanine iodide)-encapsulated mPEG-PLGA nanoparticles in normal mice and tumor-bearing mice}

To test our hypothesis that DSFNPs extravasate brain microvessels and deliver DSF across the BBB, the BBB-impermeable NIR fluorescent dye HITC was encapsulated into mPEG-PLGA nanoparticles and set up a noninvasive whole-animal fluorescence imaging method to trace the in vivo fate of the nano-formulation. The HITC dye is highly hydrophobic and was encapsulated similarly to DSF. Both the HITC dye and HITC dye encapsulated nanoparticles (HITCNPs) were administered intravenously to the normal mice (CD-1) through tail vein injections and allowed to circulate for up to $24 \mathrm{~h}$. Figure 5 shows the real-time in vivo bio-distribution for HITC and HITCNPs in live animals. From the IVIS analysis, it is clear that HITCNPs penetrated the brain at $1 \mathrm{~h}$ and reached highest levels at $24 \mathrm{~h}$. As, the free dye gets quickly removed from the body, and mice showed lower systemic fluorescence intensities compared to HITCNPs, and the difference was more pronounced at the $24 \mathrm{~h}$ time-point. Therefore, it is perceivable that a similar mechanism can be exploited for the entry of DSF to the brain at therapeutic doses. Next, we investigated the selective intratumoral accumulation of mPEG: PLGA nano formulation in tumor-bearing immunocompromised nude $(n u / n u)$ mice. When the HT29 tumor volume was around $100 \mathrm{~mm}^{3}$, mice were injected intravenously with the HITC dye alone and HITCNPs through the tail vein. Similar to the CD-1 mice, a strong NIR fluorescent signal coming from the cingulate cortex of mice brain was observed at $24 \mathrm{~h}$ after intravenous injection with the HITCNPs (Figure 6B). Brains from control mice treated with HITC alone displayed no significant
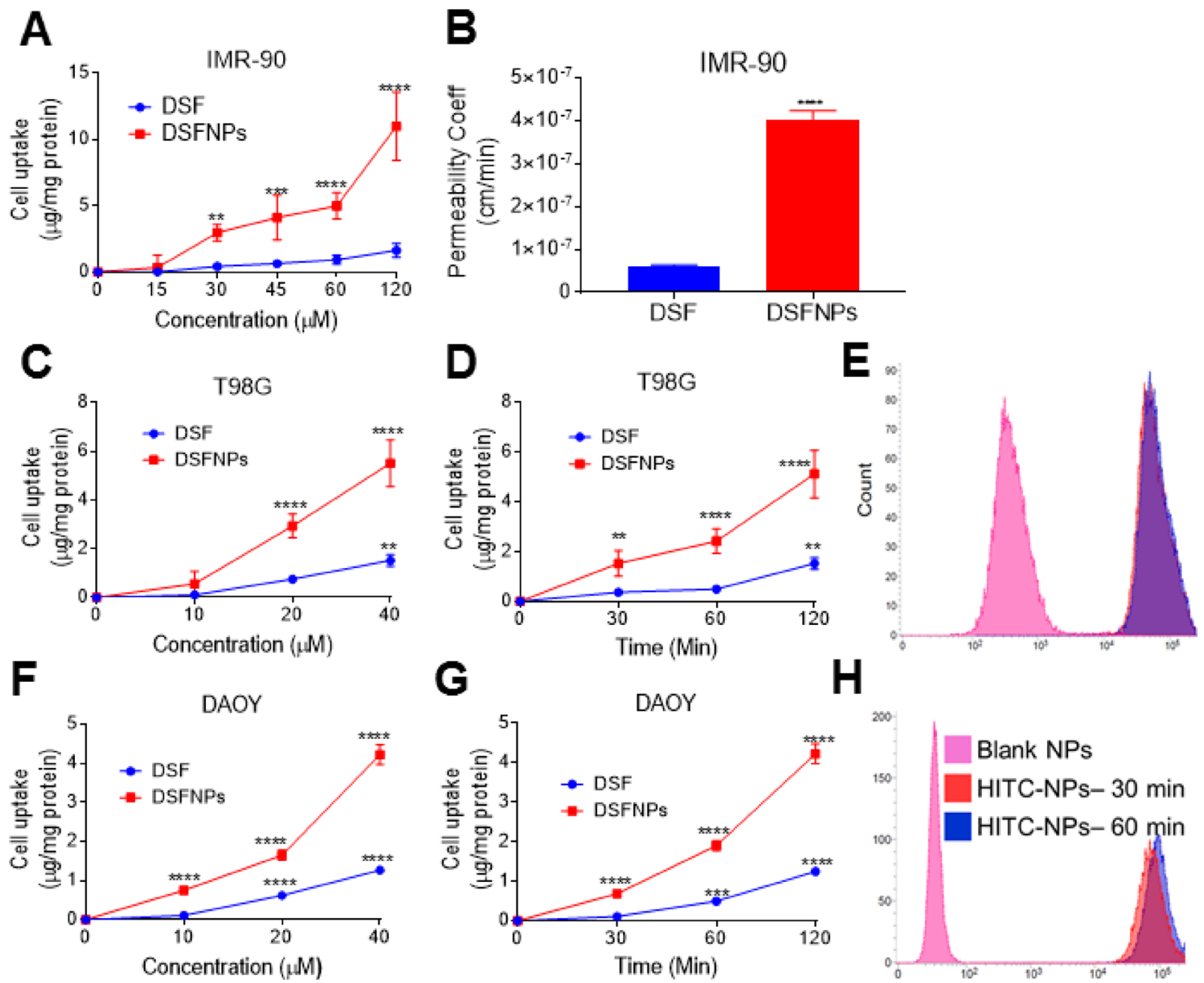

Figure 4: In vitro permeability and cellular uptake studies of DSFNPs (A) The permeability studies of DSFNPs through the IMR90 stem cells characterized as an in vitro BBB model (B) permeability coefficient of DSF and DSFNPs in IMR-90 cells; (C, D) The cellular uptake of DSF and DSFNPs in T98G cells and $(\mathbf{F}, \mathbf{G})$ DAOY cells. T98G and DAOY Cells were treated with varying concentrations of DSF or DSFNPs for $2 \mathrm{~h}(\mathbf{C}, \mathbf{F})$ or $25 \mu \mathrm{g} / \mathrm{mL}$ of DSF or DSFNPs for various times (D, G) DSF was extracted and quantified by HPLC and normalized to the protein content. (E, H) Incubation of cells with HITC-iodide dye encapsulated nanoparticles and a further measurement of fluorescence using FACS was performed. The FACS profiles and the peak shifts shown for both the T98G and DAOY cells confirmed the uptake of NPs. Data represent mean \pm SD. Experiments were performed in triplicate $(n=3)$. ${ }^{*},{ }^{* *},{ }^{* * *},{ }^{* * * *}$ indicate $p<0.05,<0.01,<0.001$ and $<0.0001$, respectively one-way ANOVA followed by Dunnett multiple comparison test. 
A

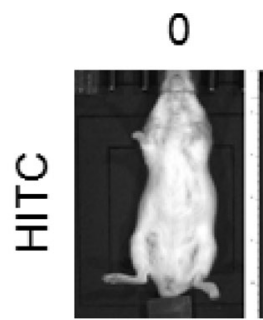

$10 \mathrm{~min}$

$1 \mathrm{~h}$

$6 \mathrm{~h}$

$12 \mathrm{~h}$

$24 \mathrm{~h}$
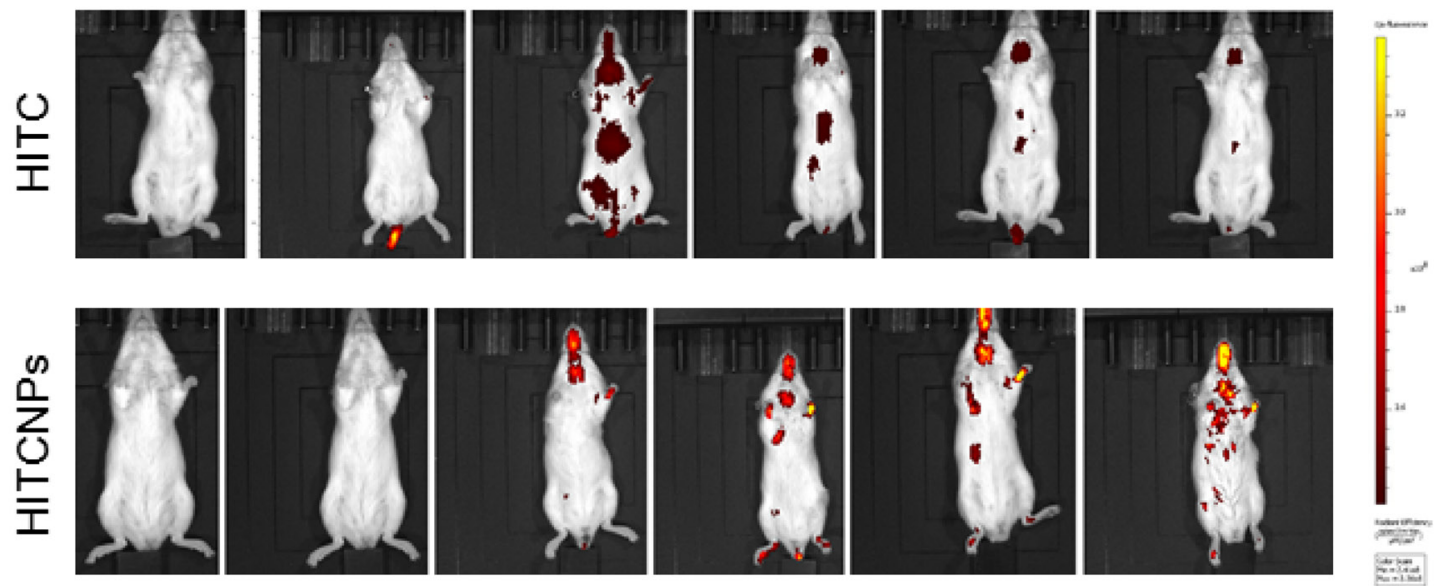

B

Dorsal

Ventral

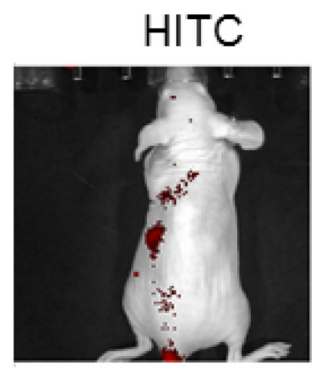

\section{HITCNPs}

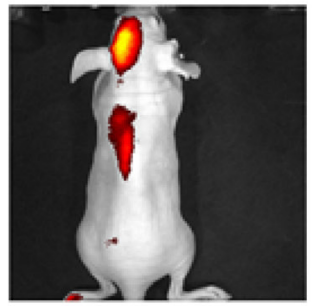

C

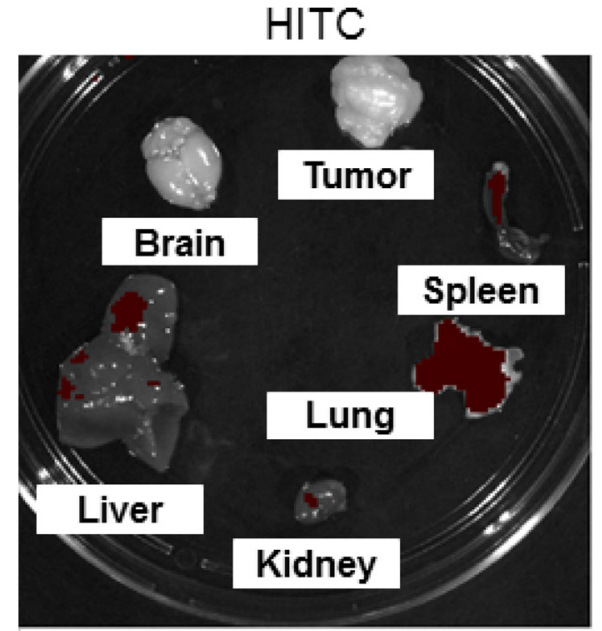

HITC
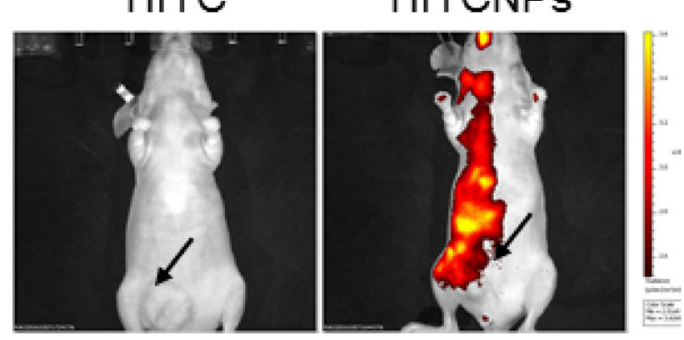

HITCNPs

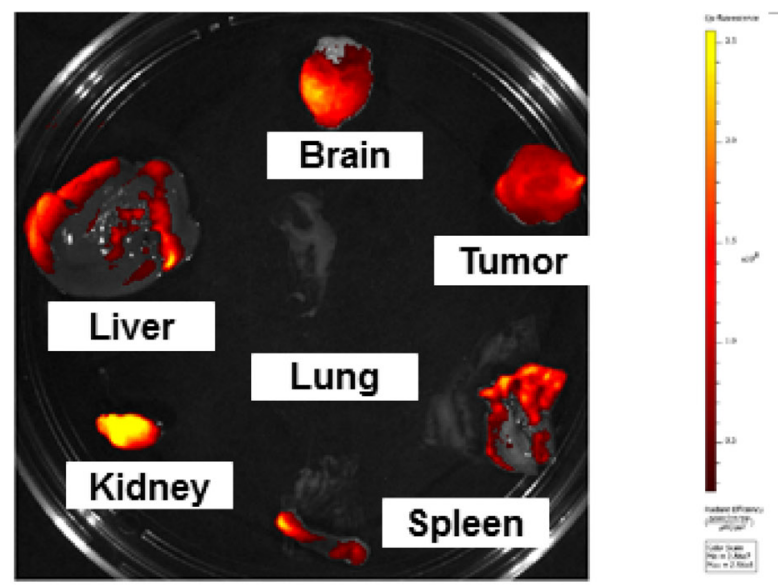

Figure 5: Real-time imaging of HITC labeled PEG-PLGA NPs (HITCNPs) in mice shows a preferential accumulation of particles in the brain. (A) To study the fate of the NPs, normal mice (CD-1strain) were injected with the near infra-red (NIR) dye HITC alone or mPEG-PLGA nanoparticles labeled with HITC. The particle distribution as visualized by imaging the animals using a Calipers In vivo Imager. The representative photographs show that there was a time-dependent and preferential accumulation of NPs in the brain. Please note the yellow color represents the highest fluorescence intensity and the dye injected alone was eliminated over the time course. (B) The HITC-loaded NPs were also injected into a nude mouse bearing a subcutaneous HT29 colon tumor on the left flank. Again, a significant accumulation of the NPs in the tumor and the brain was evident. The yellow glow indicates the highest fluorescence localization. The subcutaneous tumor was generated on the ventral side of the animal and note the accumulation of HITCNPs therein. (C) Ex vivo fluorescence intensities of various organs from the nude mice injected with HITC -labeled NPs $24 \mathrm{~h}$ post-injection. The mice were euthanized, and the vital organs were extracted, washed twice with PBS, blotted dry and transferred to a petri dish. Ex vivo organ fluorescence intensities were recorded with an IVIS at similar imaging settings. The fluorescence associated with the liver, brain, tumor, lung, kidney, and spleen is well represented. All images are displayed on the same scale and the living imaging software was used to analyze the imaging data generated as described in Methods. 
signal. Also evident is the accumulation of HITCNPs in the subcutaneous tumor formed on the abdominal ventral surface (Figure 6B right panel). To confirm the fluorescence signal we observed was truly coming from the brain and tumor, the mice were sacrificed $24 \mathrm{~h}$ - post injection and brain, tumor, and other major organs were dissected for ex vivo fluorescence imaging (Figure 6C). Organs with the brightest yellow color represent the highest HITCNPs accumulation. A strong fluorescence signal observed in excised brain and tumor of the HITCNPs treated mice confirmed that our nanoparticles formulation could cross the BBB, as well as selectively accumulate in the tumor microenvironment.

\section{DSFNPs enter the cells through clathrin- mediated endocytosis}

Next, we attempted to study the mode of entry of our formulation into the cancer cells using the HITCNPs which mimic the DSFNPs. Receptor-mediated endocytosis (RME), also called clathrin-mediated endocytosis (CME) is one of the major means of transport of drugs or drugloaded nanoparticles across the biological membranes. Further, CME reduces the non-discriminate uptake of toxic agents as well as enhances drug accumulation at the target site. NPs taken up by clathrin-dependent RME are typically destined for lysosomal degradation; whereas, clathrin-independent RME internalization leads to endosomal accumulation and sorting to a non-degradative path. After lysosomal degradation of the formulated polymer, drugs will be released into the cytosol through alterations of the lysosomal membrane integrity.

To confirm the clathrin-mediated endocytosis, we inhibited the CME by pretreatment of cells with well-established CME inhibitors chloroquine (CQ) or monodansyl cadaverine (MDC) followed by treatment with fluorescent dye loaded HITCNPs and stained with the LysoTracker. Blocking CME pathway resulted in reduced accumulation of HITCNPs as indicated by no colocalization of HITCNPs with lysosomal marker compared to $\mathrm{CQ}$ and MDC untreated cells (Figure $6 \mathrm{~A})$. Further, we observed a significant colocalization (represented with arrow marks) of HITCNPs with the lysosomes after $15 \mathrm{~min}$ incubation, and the fluorescence intensity was maintained as a function of time (15 min$4 \mathrm{~h}$ ) as shown in Figure 6B.

Further, we studied the release of DSF from the formulation and its accumulation in mitochondria. The polymer usually gets degraded in the hostile lysosomal environment, releases the DSF, which in turn is discharged into cytosol through lysosomal rupture. Disruption of lysosomal integrity was examined by measuring immunofluorescence associated with LAMP1, a lysosomal membrane protein that protects lysosomes from the acidic hydrolases. After treatment with DSFNPs in T98G cells, a decreased LAMP1 immunofluorescence was observed when compared to the untreated cells indicating the loss of lysosomal membrane integrity (Figure 6C). Next, we found that the released HITC was selectively recruited to the mitochondria, which was confirmed by the colocalization of HITC fluorescence with DiOC6(3), a green fluorescent dye that selectively stains the mitochondria (Figure 7). The mitochondrial accumulation was highest at the $4 \mathrm{~h}$ time-point as indicated by a strong merging of fluorescence of HITC, and the mitochondrial tracker dye. Taken together these results confirm that mPEGPLGA nano formulation undergoes CME, accumulates in lysosomes, upon polymer degradation, the drug will be released into the cytosol followed by its mitochondrial uptake.

\section{DSFNPs induce oxidative stress}

Disulfiram, as a free drug, has been reported to induce ROS [32] and activate the MAPK pathway [2]. Therefore, we tested the ability of DSFNPs to induce ROS by staining the T98G and DAOY brain cancer cells with DCF-DA $\left(2^{\prime}, 7^{\prime}\right.$-dichlorofluorescein diacetate) or DHE (dihydroethidium). DCF-DA is a fluorogenic dye that measures the activity of peroxyl and hydroxyl radicals in the cells. The cell-permeable DCF-DA is deacetylated by cellular esterases to release a non-fluorescent intermediate, which upon interaction with ROS is oxidized to yield fluorescent DCF [32, 33]. DHE is a superoxide indicator, which exhibits blue- fluorescence in the cytosol until oxidized by superoxides, after oxidation it intercalates within the cellular DNA and stains the nucleus with a bright fluorescent red color with 500 and $580 \mathrm{~nm}$ excitation and emission wavelengths respectively.

Microscopic examination of DCF-DA and DHE stained DSF or DSFNPs treated cells demonstrated a significant increase in the intensity of DCF and DHE staining as shown in Figure 8A, 8C respectively and Supplementary Figure 4A, 4C respectively. FACS analysis further confirmed a significant increase in ROS levels in drug-treated T98G and DAOY cells as shown in Figure 8B, 8D, and Supplementary Figure 4B, 4D. NAC pretreatment quenched and reversed these effects confirming the DSF mediated ROS elevation. $\mathrm{H}_{2} \mathrm{O}_{2}$ and antimycin were used as positive controls as peroxide and superoxide inducers respectively. These results demonstrate that DSF retains its oxidative stress-inducing ability after its formulation into DSFNPs. Further, DSFNPs exhibited superior oxidative stress induction when compared to the free DSF in both T98G and DAOY cells.

\section{DSFNPs induce loss of mitochondrial membrane integrity}

It is well established that mitochondria play a major role in cell signal transduction through ROS production [32] and activating several stress-responsive pathways [33-35] 

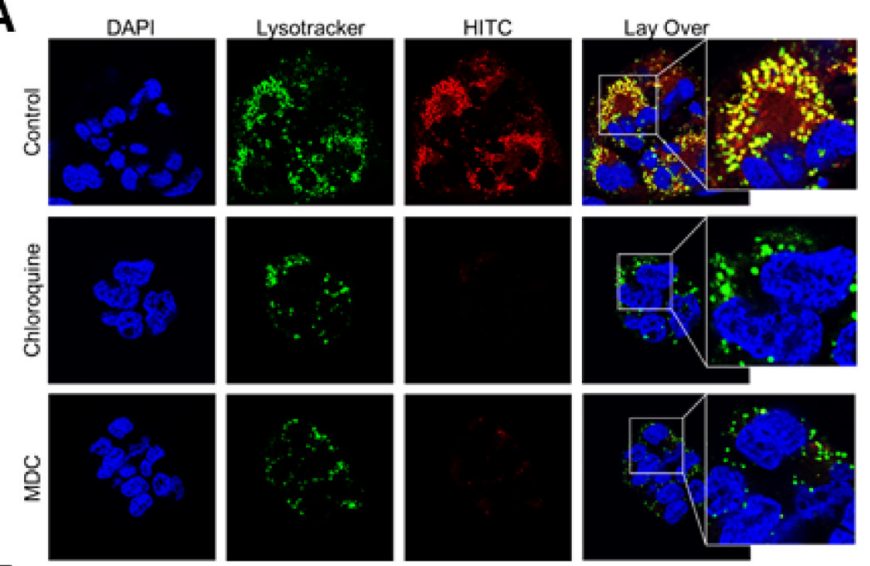

B
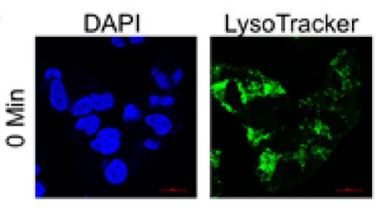

HITC
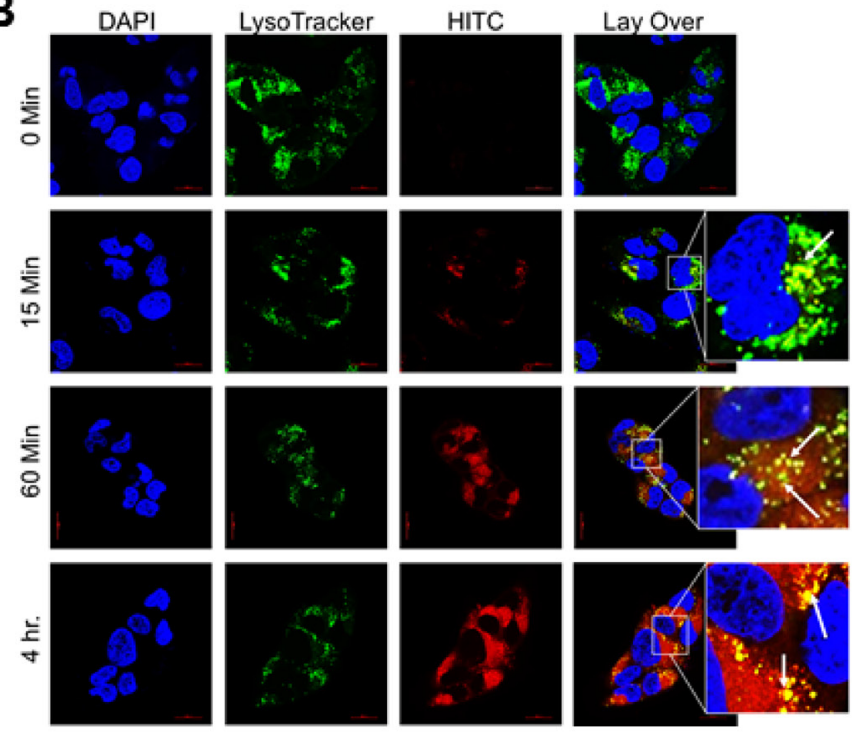

C
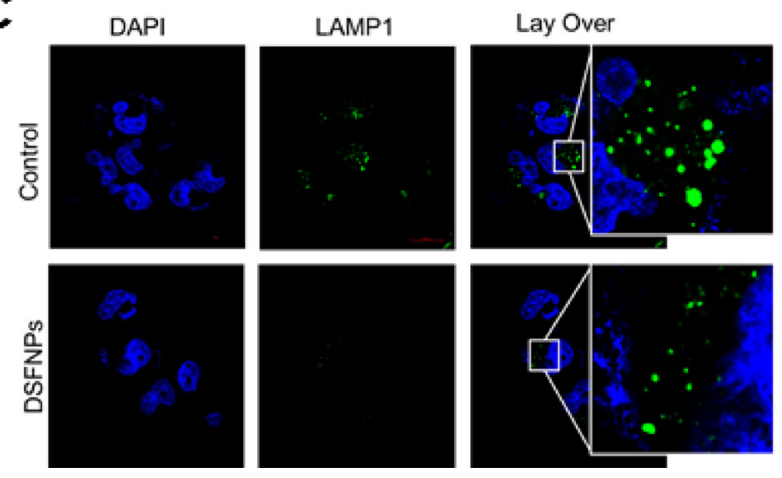

Figure 6: Clathrin-mediated endocytosis of HITCNPs and time-dependent accumulation of HITC-NPs in lysosomes in glioblastoma cells and evidence that loss of lysosomal membrane integrity mediates the drug release. (A) The confocal photomicrographs show that following cellular internalization, the HITC-labeled NPs colocalize with the lysosomes in T98G cells. The first panel represents the cells treated with HITCNPs alone (red fluorescence) and next two represent cells pre-treated with chloroquine and MDC followed by incubation with HITCNPs. The cells were stained with the LysoTracker (green fluorescence), a marker for lysosomes. The overlaid confocal microscopy images show the colocalization of the lysosomal marker and the HITCNPs, represented by a yellow-orange punctate pattern. Note that both chloroquine and MDC strongly inhibited the internalization of DSFNPs. (B) Increased co-localization of HITC-NPs in lysosomes in T98G cells in a time-dependent manner. Lysotracker was used to label the lysosomes. A clear colocalization of the HITC-loaded NPs (red) with the lysosomes (green) is evident in the overlaid images showing a yellow-orange punctate pattern. (C). The decrease in the expression of lysosomal membrane protein LAMP1 in T98G cells treated with DSFNPs. Cells were stained with antibodies to LAMP1, a lysosomal membrane protein degraded by acid hydrolases. Decreased LAMP1 staining in DSFNP-treated cells is apparent. 
leading to autophagy and apoptotic cell death [36, 37]. Loss of mitochondrial transmembrane potential affects the membrane integrity, and it releases ROS and triggers the initiation of the apoptotic cascade [37, 38]. Consequently, the apoptotic inducers such as AIF (apoptosis-inducing factor) and cytochrome $\mathrm{C}$ leak out through the damaged mitochondrial membrane and translocate into the nucleus to trigger the apoptotic chain of events $[39,40]$. To establish the effect of DSF-induced ROS on mitochondria, we pretreated T98G and DAOY cells with cyclosporine-A. After $4 \mathrm{~h}$, they were exposed DSFNPs for one day and then stained with DCF-DA or DHE (dihydroethidium). Cyclosporine $\mathrm{A}$ is an established inhibitor of mitochondrial permeability transition [40] (MPT) pore by binding with cyclophilin D $[41,42]$ and thereby upholds the integrity of the mitochondrial membrane. We observed a strong decrease in the fluorescence intensity of DCF-DA and DHE in cyclosporine-A pretreated cells suggesting that ROS induced by DSF were significantly diminished, whereas, in the absence of cyclosporine A, the cells showed intense fluorescence with both free-radical scavengers. These observations encouraged us to probe if the mitochondrial membrane was depolarized by DSFNPs to release ROS. To confirm this, we adopted a flow cytometric method for the quantitative measurement of mitochondrial transmembrane potential $\left(\Delta \Psi_{\mathrm{m}}\right)$ using 3,3'-Dihexyloxacarbocyanine Iodide $\left[\mathrm{DiOC}_{6}(3)\right]$. We used carbonyl cyanide m-chlorophenylhydrazone (CCCP), a potent mitochondrial oxidative phosphorylation uncoupler, which renders the mitochondrial inner membrane permeable to protons as a positive control for membrane depolarization. We costained the cells with DHE to correlate DSF-induced ROS and decrease in the mitochondrial membrane potential $\left(\Delta \Psi_{\mathrm{m}}\right)$. In this study, we have observed a significant decrease in mitochondrial trans-membrane potential in DSFNPs treated cells (T98G and DAOY cells) when compared to the untreated cells (Figure 8E, 8F). These results were further validated by employing negative and positive controls of oxidative stress, N-acetylcysteine (NAC) and antimycin A respectively. NAC pretreatment diminished the effect of DSFNPs on mitochondrial membrane depolarization, and antimycin potentiated the DSFNPs effect. Taken together, these data clearly demonstrate the interlink between DSF induced ROS and loss of mitochondrial
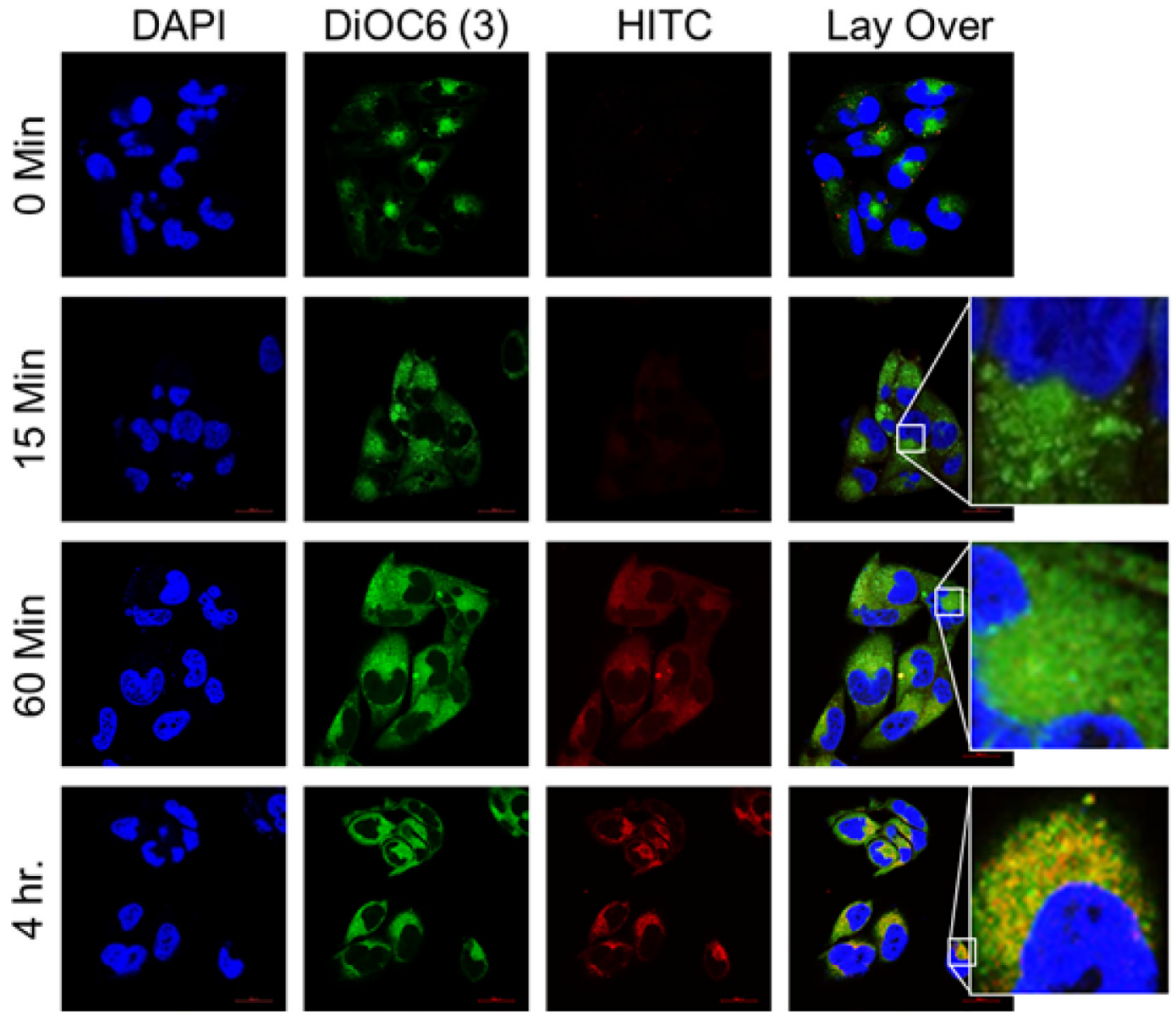

Figure 7: Co-localization of HITCNPs with mitochondria. (A) Increased accumulation of HITCNPs in mitochondria in T98G cells in a time-dependent manner. Cells were treated with the DiOC6(3) and HITC-labeled nanoparticles as described in Methods. The overlaid images show the overlap of the mitochondrial membrane marker (DiOC6(3), green) with that of HITCNPs (red), as denoted by the yellow-orange punctate pattern, which is particularly strong at $4 \mathrm{~h}$ post-treatment. 
membrane potential, which leads to increased leakiness of mitochondria.

\section{ROS induced by DSFNPs activate the MAPK pathway}

Mitogen-activated protein kinase (MAPK) signaling pathway is well known to play a critical role in diverse cellular processes including the regulation of endoplasmic reticular stress, cell proliferation, differentiation, autophagy [43] and apoptosis [2]. Further, it is well-known that reactive oxygen species (ROS) are potent activators of the c-Jun N-terminal kinase (JNK) through oxidative inactivation of endogenous JNK inhibitors, such as JNK phosphatases and GST-pi [42-44]. Figure 9A represents the effect of DSF and DSFNPs on the activation of the
A

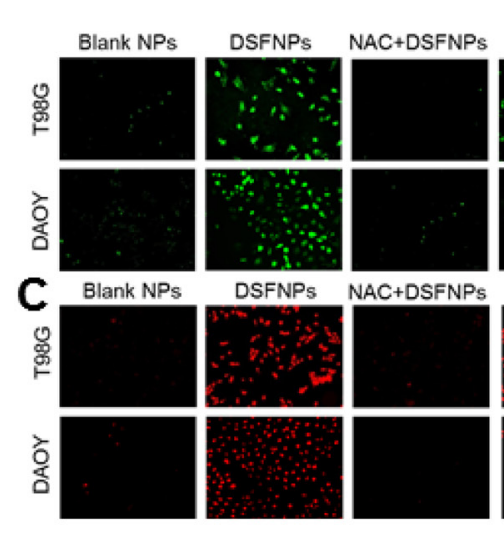

E

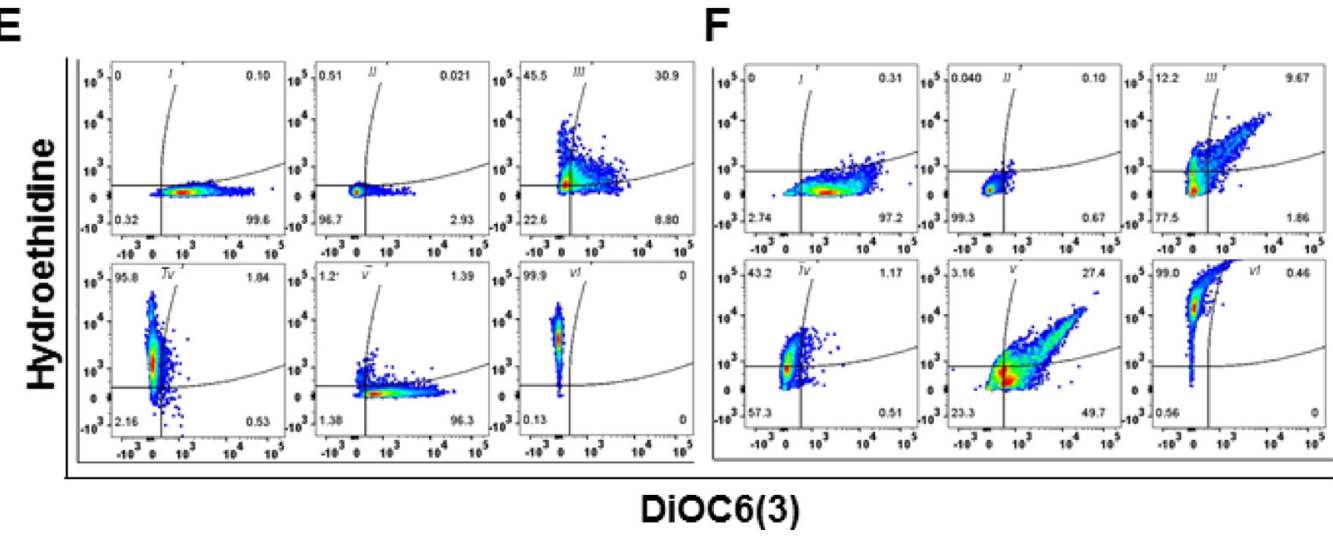

B
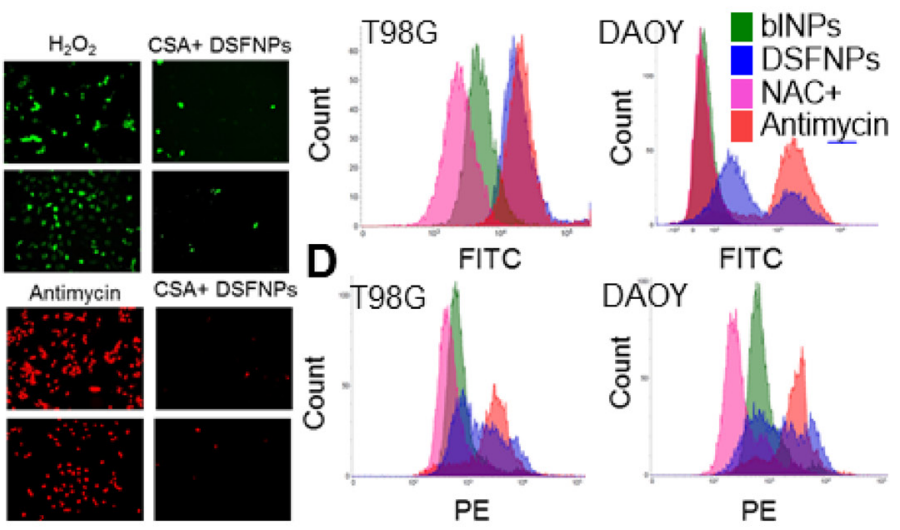

$\mathbf{F}$ 
MAPK pathway. Though the basal protein expression of JNK, P38, and ERK1/2 was not affected much by the treatment, the expression of phosphorylated JNK, P38 and ERK1/2 increased persistently and reproducibly. We observed a corresponding decrease in phosphoAKT levels. However, the AKT protein levels remained constant. We also observed a significant increase in mitochondrial stress-sensitive protein CHOP.

The relationship between MAPK pathway and AKT is intriguing. Even the role of AKT in apoptosis is confusing. Activation (phosphorylation) of AKT renders tumor cells resistant to several chemotherapeutic drugs $[45,46]$. It is reported that AKT activation occurs as a transient response of the cells undergoing apoptosis as a self-defense mechanism, being a pro-survival marker. However, an eventual decrease in AKT phosphorylation may prove necessary, for the overall induction of apoptosis [47].

\section{DSF induces nuclear translocation of apoptosis- inducing factor (AIF)}

The above observations clearly suggest a role for the operation of apoptotic pathways in mediating the DSF cytotoxicity. It was reported that DSF inhibits caspase family proteins [46], on the other hand, we observed upregulation of proapoptotic proteins and downregulation of antiapoptotic proteins (Figure 9B), which strengthened the observation of Carmody et al. [47] and prompted us to check expression and localization of caspaseindependent mitochondrial apoptotic protein such as the AIF [48-50]. Immunofluorescence microscopy revealed the subcellular co-localization of AIF with the nucleus in the T98G cells treated with DSFNPs for $24 \mathrm{~h}$ compared with the controls.

\section{DSFNPs induce apoptosis}

To determine the mechanism of cytotoxic effects observed with DSFNPs, Annexin-V/FITC apoptosis assay was performed by a flow cytometer in T98G and DAOY cancer cells. We observed a substantial increase in annexin-V positive cells with both cell lines compared to the untreated controls in concentration-dependent manner (Figure 10A).

Next, the changes in the protein expression of various pro- and anti-apoptotic proteins were quantitated by western blot analysis. DSFNPs treatment resulted in a significant increase in the expression of pro-apoptotic markers such as cleaved-PARP, NOXA, Bax proteins and corresponding decreases in the levels of pro-apoptotic proteins. Nevertheless, in agreement with the report by Nobel et al., we observed a decrease in caspase expression [46] raising the possibility of caspase-independent apoptosis mediated through mitochondria by DSFNPs (Figure 10B) [50].

\section{In vitro cytotoxicity of DSFNPs}

In continuation, the in vitro cytotoxicity of DSF and DSFNPs in various brain cancer cells were compared. Blank nanoparticles did not show any cytotoxicity among the cell lines tested (data not shown). The cytotoxic effects of DSF and DSFNPs in a timedependent manner with $24 \mathrm{~h}, 48 \mathrm{~h}$, and $72 \mathrm{~h}$ time points were determined, and the results are shown in Figure $11 \mathrm{~A}$. The $\mathrm{IC}_{50}$ values of free DSF in T98G cells were 201,122 , and $30 \mu \mathrm{M}$ and in DAOY cells were 51, 11 and $9 \mu \mathrm{M}$ at 24,48 , and $72 \mathrm{~h}$, respectively. The $\mathrm{IC}_{50}$ values of DSFNPs in T98G cells were 167, 39 and $18 \mu \mathrm{M}$ and in DAOY cells were 34, 6 and $3 \mu \mathrm{M}$ at 24,48 and 72 $\mathrm{h}$, respectively. The $\mathrm{IC}_{50}$ values of DSF were slightly higher compared to DSFNPs, and the difference in $\mathrm{IC}_{50}$ between DSF and DSFNPs increased over the time from 24 to $72 \mathrm{~h}$ indicating the sustained release of DSF. We have already shown that DSF, as a free drug can quickly inactivate the MGMT DNA repair in xenografted brain tumors [10]; it should be noted that DSFNPs function as a drug reservoir and a long-term supply is expected to augment the antitumor efficacy. The cytotoxic profiles of DSFNPs against other brain cancer cells were as represented in Figure 11B.

In agreement with the extent of cytotoxicity noted above, the clonogenic survival assays in both T98G and DAOY cells revealed a similar trend of cell killing by DSFNPs, at an $\mathrm{IC}_{20}$ concentration. The decrease in the number of colonies was statistically significant with increased concentrations of free and encapsulated DSF, though, the differences between them were marginal (Figure 11C).

\section{Demonstration of in vivo efficacy of DSFNPs in an intracranial medulloblastoma xenograft model}

To evaluate and compare the anticancer efficacy of DSFNPs versus the free DSF in an orthotopic brain cancer xenograft setting, we first used lentiviral transfection of luciferase and developed a DAOY human medulloblastoma cell line with a high level of stable luciferase expression. These cells were implanted by stereotaxic injection into mice brains to generate intracranial xenografts and the tumor growth was assessed by quantitative bioluminescence using an in vivo imaging system. The tumor-bearing animals were divided into four groups and received just the vehicle, blank nanoparticles, DSF or DSFNPs. DSF (30 mg/kg) or equivalent amount of DSFNPs were administered i.p. three times a week for 5 weeks and the results are shown in Figure 12. The representative color luminescent images representing the spatial distribution of photon counts in the brain shows that disulfiram alone or blank nanoparticles did not affect the luciferase expression 
on day 35 of treatment, however, the DSFNPs, on the average showed a marked loss of bioluminescence (Figure 12A). The quantitative changes in average bioluminescence radiance calculated from 6 animals over the therapy period are drawn in Figure 12B; DSF, in its unencapsulated form, induced only a marginal, but discernible effect on tumor growth on days 28 and 35 .
In contrast, the DSFNPs elicited a strong and significant tumor growth inhibition, with a maximal 7-fold more tumor cell killing than the free drug during the same period. The relative $\%$ decrease in bioluminescence units shown in Figure 12C provides a better representation of the antitumor effects observed in our study. Compared to DSF, DSFNPs were 2.5, 4.6 and 7.5 times more potent
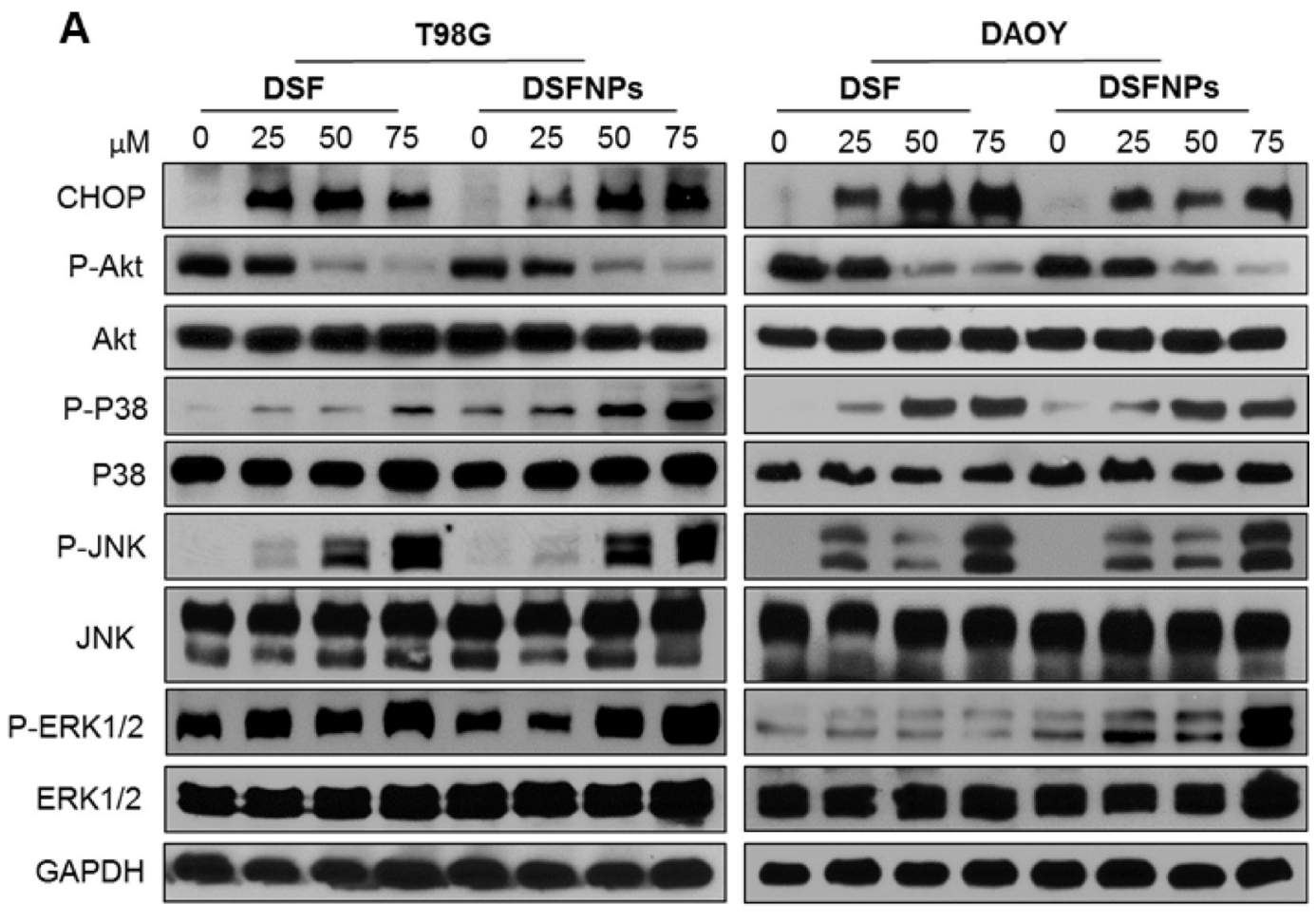

$\mathbf{B}$
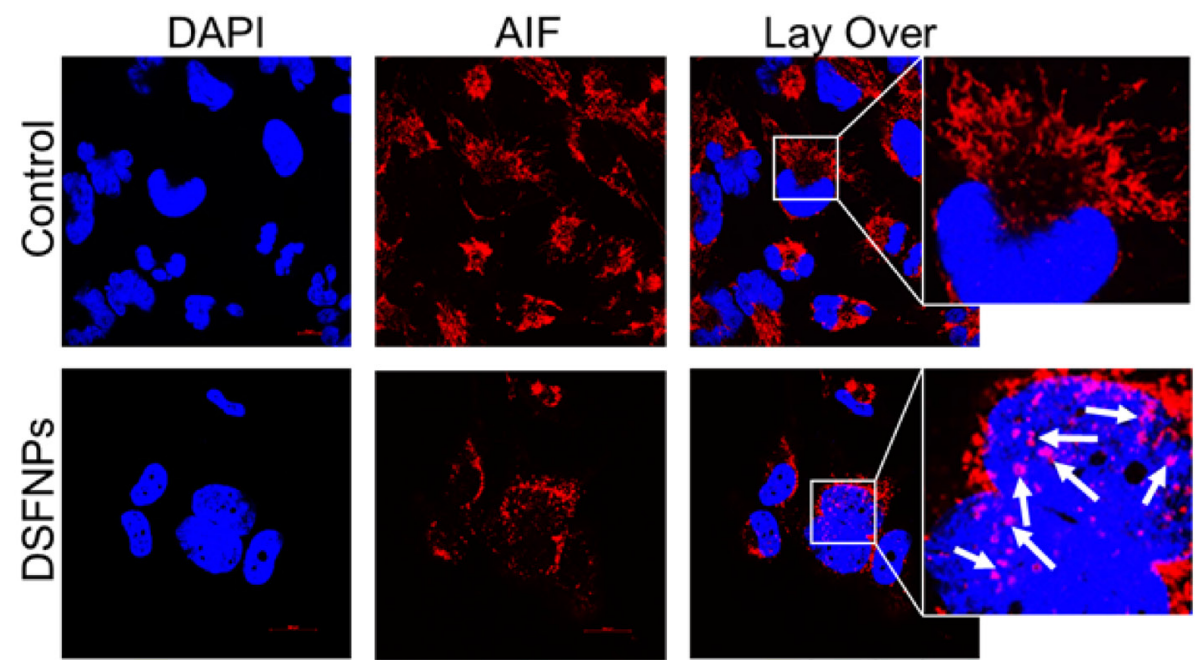

Figure 9: DSFNPs induce MAP-Kinase pathway components and nuclear translocation of AIF (apoptosis-inducing factor). (A) Cells were treated with DSF or DSFNPs at different concentrations for $24 \mathrm{~h}$ and western blotting using specific antibodies was performed. Both the DSF and DSFNPs increased the expression of CHOP and phosphorylation of ERK, p38, and JNK and blocked the phosphorylation of AKT in a concentration-dependent manner. GAPDH was used as loading control. (B) Nuclear translocation of AIF in T98G cells induced by DSFNP treatment for $24 \mathrm{~h}$. Cells were analyzed by confocal microscopy after immunostaining with antibodies to AIF. Arrows indicate the translocation AIF into the nucleus. AIF is a mitochondrial protein and migrates into the nucleus to initiate the caspase-independent apoptosis by causing DNA fragmentation and chromatin condensation. 
in inhibiting the tumor growth on days 21, 28 and 35 respectively. These kinetics also suggest that DSF or DSFNPs as a single agent are likely to exert a delayed antitumor effect and extended cycles of treatment may be needed. There were no significant changes in the average body weight of the mice at the given dose in any group, suggesting that the treatment did not exert undue adverse effects to the host organs (Figure 12C). Collectively, the xenograft study justifies our objective of encapsulating the DSF in nanoparticles for brain tumor therapy.

\section{CONCLUSIONS}

Current therapies for malignant brain tumors are not effective, and an urgent need exists for designing improved treatment strategies. In this study, a nanoformulation containing DSF was successfully prepared using mPEGPLGA as polymer, and the formulation was optimized to achieve therapeutically-relevant parameters, including a lower particle size, good polydispersity index and higher entrapment efficiency. The DSFNPs were further

A

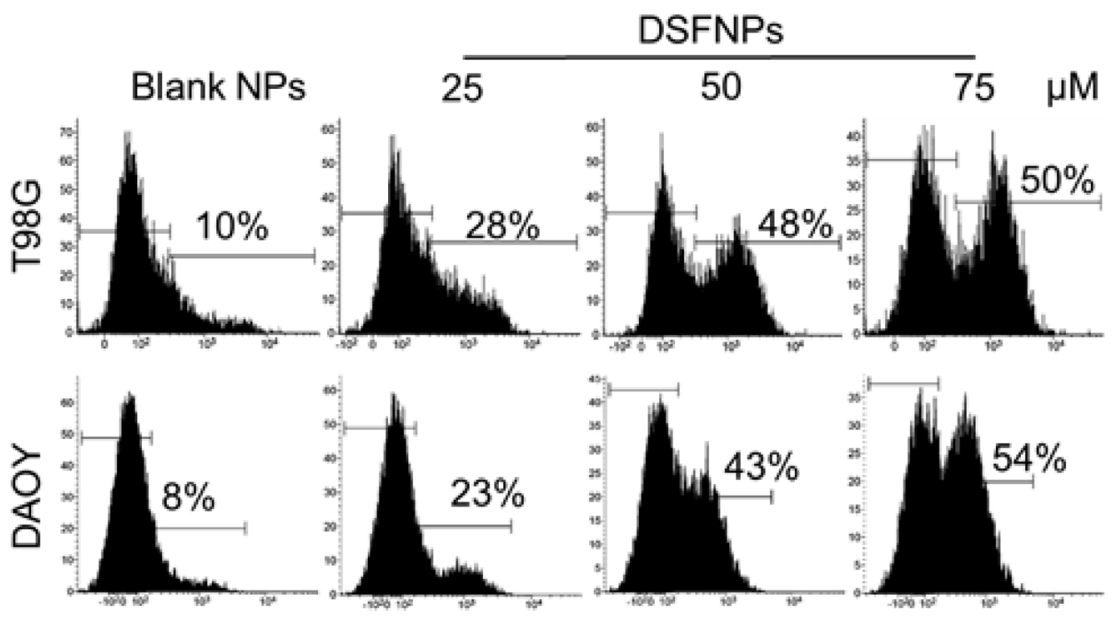

B
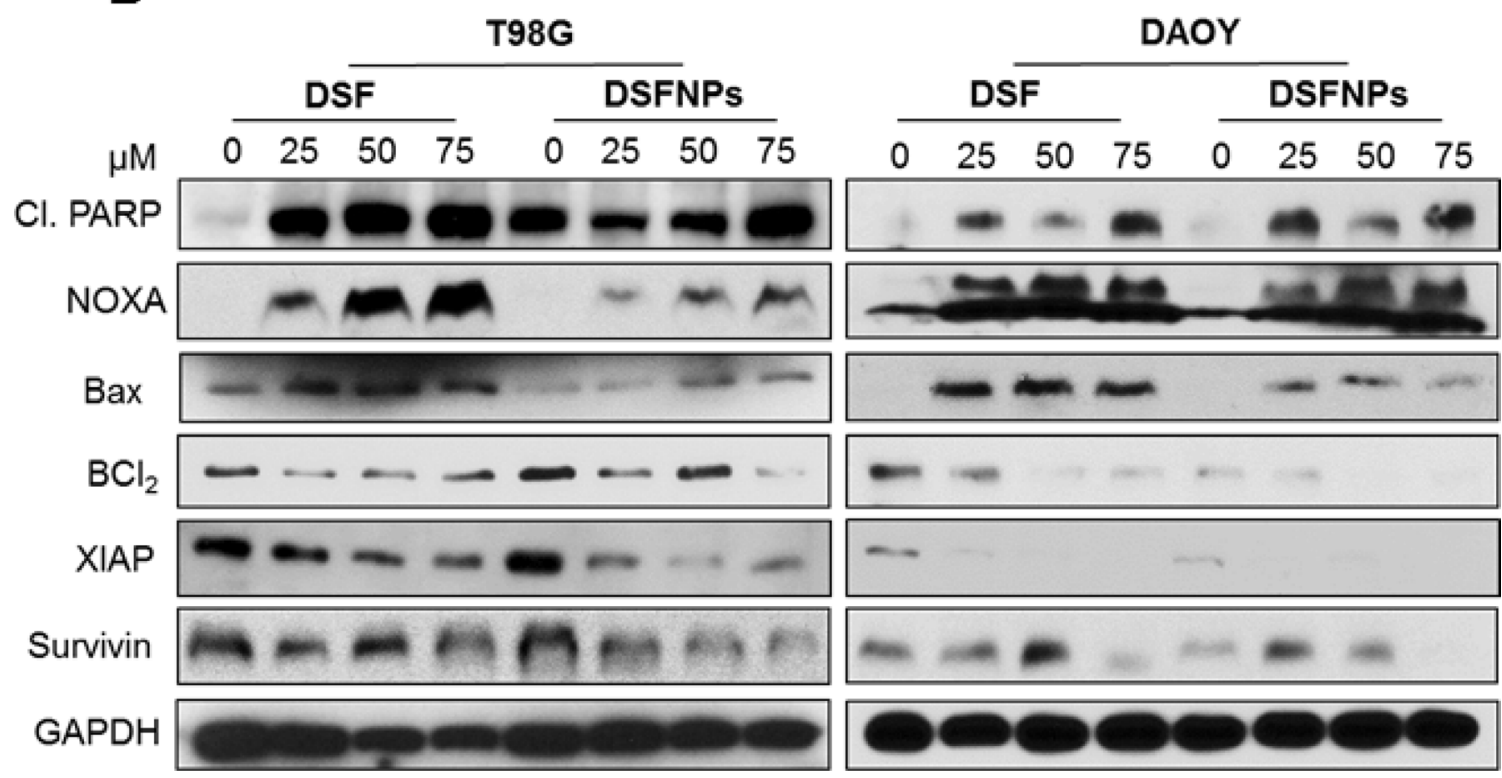

Figure 10: DSFNPs trigger apoptosis in brain tumor cells. (A) Flow cytometric histograms of T98G and DAOY cells stained with FITC-annexin V after treatment with DSFNPs are shown. The shift in the peak to the right indicates the increase in the apoptotic cells in a concentration-dependent manner. The $\%$ of annexin-positive cells in the early-apoptotic phase are indicated. Annexin $\mathrm{V}$ is a member of the annexin-family of proteins that binds to phosphatidylserine in early-apoptotic cells. FITC-conjugated annexin V was used to detect the apoptosis using flow cytometry. Increase in the fluorescence indicates the increased number of the apoptotic cell population. (B) Expression of apoptotic and anti-apoptotic regulatory proteins after DSF or DSFNP treatment. Cells were treated with DSF and DSFNPs at different concentrations followed by western blotting. Both the free and encapsulated drug increased the expression of apoptotic proteins (cleaved PARP, Bax, and NOXA) and decreased the expression of anti-apoptotic proteins ( $\mathrm{BCl}_{2}, \mathrm{XIAP}$, and Survivin) in a concentration-dependent manner. GAPDH was used as loading control. 
characterized for the surface, physical and thermal properties along with stability, permeability and drug uptake using appropriate techniques. A near-infrared dye encapsulated formulation with similar characteristics as that of DSFNPs revealed that the formulation selectively accumulates in the brain and in subcutaneous xenografted tumors. These results suggested that the formulation was able to efficiently deliver DSF to the brain with an increased plasma half-life. While the smaller size of the particles may facilitate the transport across the BBB, an enhanced permeability retention in the tumor vasculature $[24,54]$ may aid a preferential accumulation of these formulations in cancer tissues. A hypervascularity, incomplete vascular architecture, secretion of vascular permeability factors leading to extravasation within the malignant tissues and absence of lymphatic drainage for clearance may all contribute a greater accumulation of DSFNPs in brain cancers. The DSFNPs were able to induce killing of brain tumor cells and elicit various molecular alterations and apoptosis in a potent manner, with a marginally superior efficacy compared to the un-encapsulated free drug. However, it should be noted that disulfiram was released in a sustained fashion over a long time from the nanoparticles and in essence, the cytotoxic events observed were achieved at lower drug concentrations. In fact, the vastly superior antitumor effects of DSFNPs (compared to DSF by itself) we observed in orthotopic xenograft studies are consistent with this notion and justify the use of the nano-formulated drug in a clinical setting. Further, we were interested to see the downstream effects of DSFNPs after their entry into cells. A schematic representation of the uptake and cytotoxic mechanisms of DSFNPs in glioblastoma cells, as revealed by the current study is shown in Figure 13. We found the DSF nanoparticles to internalize through clathrin-coated vesicles, and accumulated in lysosomes and subsequently in mitochondria. Mechanistically, DSFNPs through, a release of DSF in a sustained manner induced ROS and loss of mitochondrial transmembrane potential. DSFNPs also activated the MAPK pathway and induced nuclear translocation of AIF to trigger apoptosis. DSFNPs induced
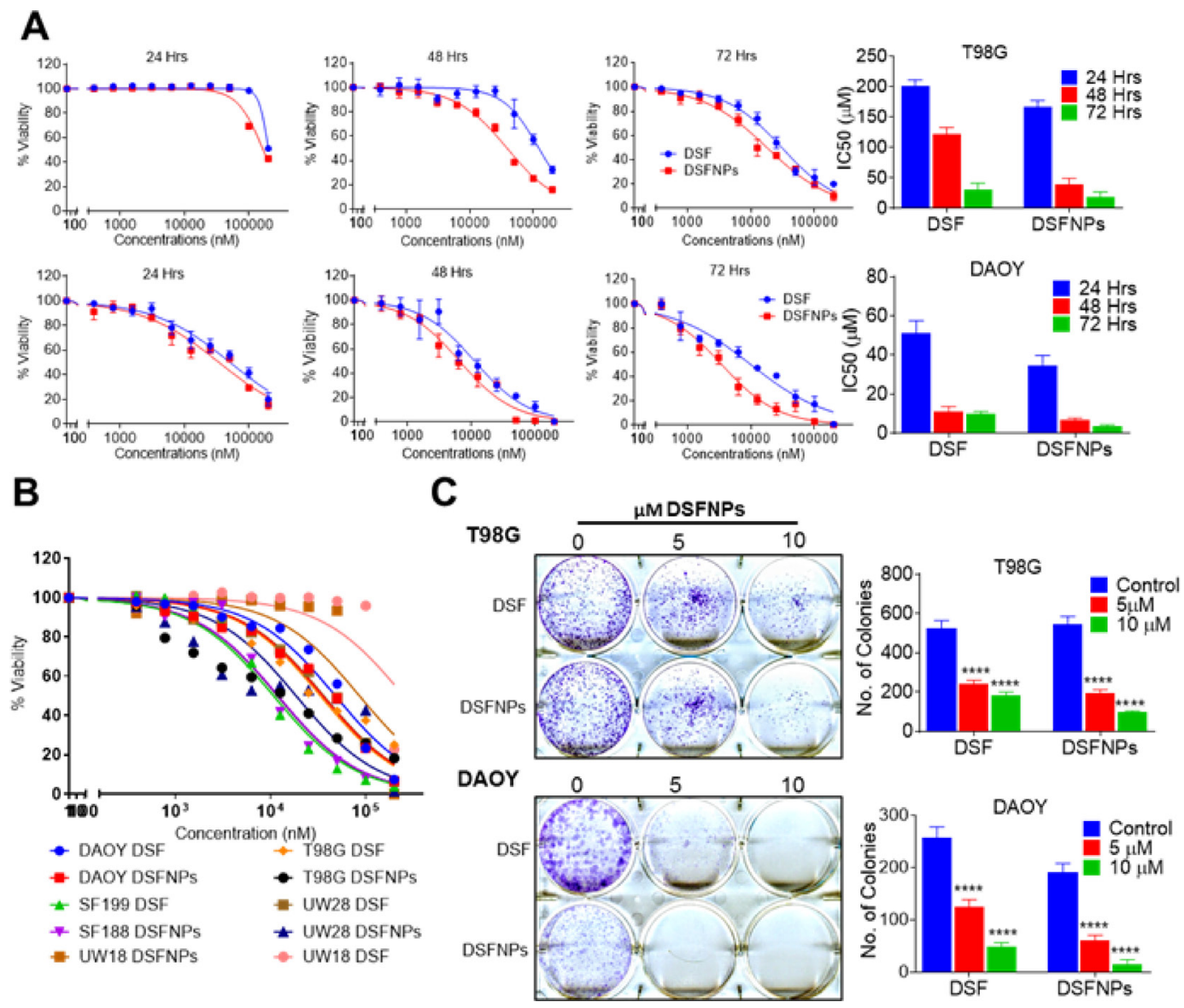

Figure 11: DSFNPs induce cytotoxicity in brain tumor cells to the same extent or more efficiently as DSF. (A) Effect of DSF and DSFNPs at varying concentrations and time in T98G and DAOY cells. (B) Cytotoxicity of DSF and DSFNPs against other brain tumor cell lines (SF188, UW28, UW18) at $72 \mathrm{~h}$. A fluorescence assay using resazurin was used. (C) The suppressive effect of DSF and DSFNPs on colony formation of T98G and DAOY cells. Representative images of the colony-forming assay are shown on the left panel. Colonies were counted using ImageJ software and plotted as bar graphs in the right panel. Data represent mean $\pm \mathrm{SD}$. Experiments were performed in triplicate $(n=3)$. ${ }^{* * * *}$ indicate $p<0.05,<0.01,<0.001$ and $<0.0001$, respectively one-way ANOVA followed by Tukey's multiple comparison test. 
cytotoxicity and exerted potent anti-clonogenic effects against T98G and DAOY cells (Figure 11). Based on these findings, studies are underway to determine the antitumor effects of DSFNPs with or without copper gluconate and temozolomide in glioblastoma orthotopic xenografts.

\section{MATERIALS AND METHODS}

\section{Cell lines and cell culture}

Human brain cancer cell lines DAOY and T98G were obtained from the American Type Culture Collection
(ATCC, Rockville, MD). T98G and DAOY cells were cultured in Dulbecco's minimum essential medium (DMEM) (Corning) supplemented with 10\% fetal bovine serum (Atlanta Biologicals Inc.) and antibiotics. All cells were cultured in a humidified atmosphere with $5 \% \mathrm{CO}_{2}$ at $37^{\circ} \mathrm{C}$.

\section{Animals}

Female CD-1 mice, female athymic nude (nu/ nu) mice, and the triple immune-deficient NCG (NOD CRISPR Prkdc I12r gamma) mice were obtained from

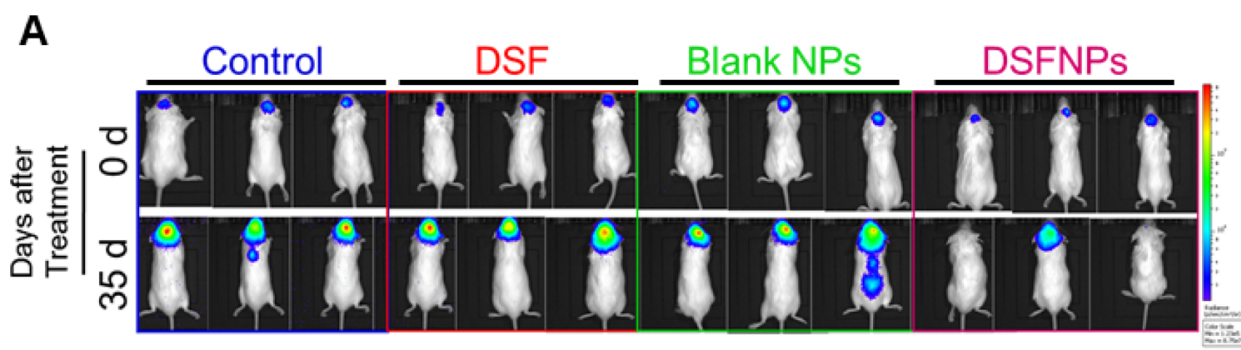

B

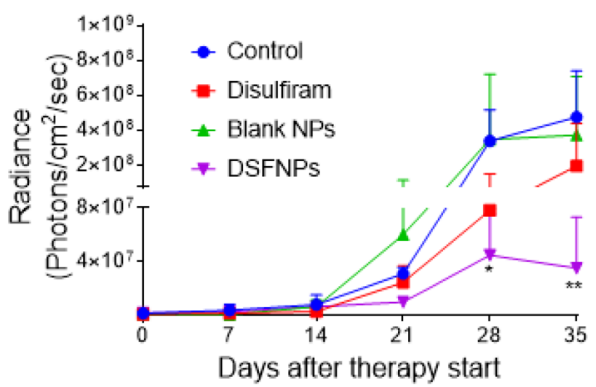

D

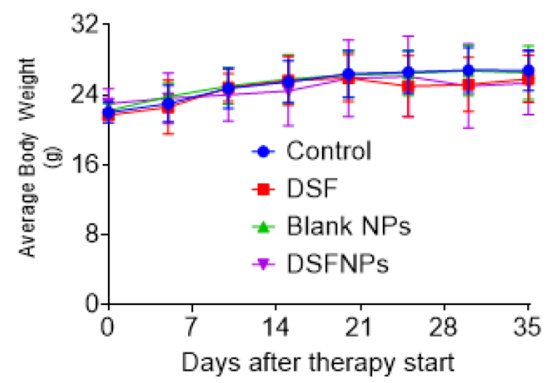

C

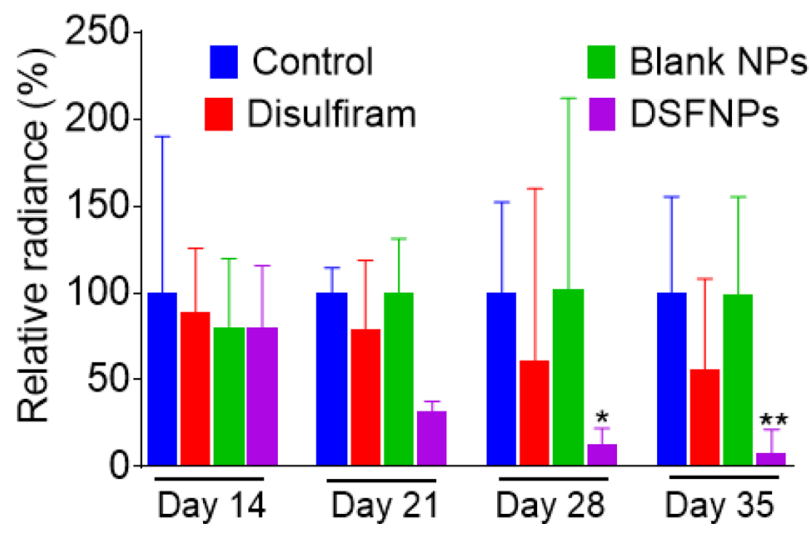

Figure 12: Strong antitumor efficacy of DSFNPs relative to DSF in DAOY human medulloblastoma intracranial xenografts. (A) Representative images of bioluminescence acquisition of brain tumor at the beginning and end of therapy. Luciferaseexpressing DAOY cells were injected into the brains of immunocompromised NCG mice as described in Methods. DSF or DSFNPs at $30 \mathrm{mg} / \mathrm{kg}$, nanoparticles without DSF loading or the vehicle alone were given to the mice i.p. three times a week, and IVIS imaging was performed once every week. The bioluminescence was compared on a common scale ranging from $1.23 \times 10^{5}$ to $8.75 \times 10^{7}$ as shown in the color intensity bar (blue to red) on the right. The results showed a significant decrease in tumor burden in mice treated with DSFNPs, but not with DSF. (B) Average time-dependent changes in bioluminescence radiance in DAOY xenografts receiving therapy over 5 weeks. The radiance was processed and computed using the LiveImage software. Data represent mean $\pm \mathrm{SD}, n=6 .{ }^{*}$ indicates significance at $p<0.05$ and ${ }^{* *} p<0.01$; one-way ANOVA followed by Dunnett's multiple comparison tests were performed. (C) Changes in relative radiance in the treatment groups over days 14 to 35 are shown in the bar graphs. The average radiance of the control group mice was considered $100 \%$ at each time point. The data are expressed as mean \pm SD. (D) The average body weights of the mice were plotted against the treatment period. 
Charles River Laboratories. All animal procedures were performed according to the Institutional Animal Care and Use Committee (IACUC) guidelines.

\section{Chemicals, antibodies, and other reagents}

Disulfiram was purchased from Sigma (St Louis, MO). The m-polyethyleneglycol-polylactic-glycolic acid (mPEG-PLGA) polymers were purchased from Advanced Polymer Materials Inc. (Montreal, Canada). Solvents were purchased form Fisher Scientific. The near-infrared dye, 1,1',3,3,3',3'- Hexamethyl indotricarbocyanine iodide (HITC), 2', 7'-dichlorofluorescein diacetate (DCFDA), chloroquine, cyclosporine A (CSA) and rest of the chemical reagents were purchased from Sigma (St Louis, MO). All antibodies were purchased from Cell Signaling Technology (Danvers, MA). Dihydroethidium (DHE), 4,5-dihydroxy-1,3- benzenedisulfonic acid disodium salt monohydrate (tiron), N-acetyl cysteine (NAC), 3,3'- Din-hexyloxacarbocyanine iodide (DiOC6(3)) and carbonyl cyanide 3-chlorophenyl hydrazine (CCCP) were from Thermo Fisher. The concentrations of the above reagents used for the study were $5 \mu \mathrm{M}$ DHE, $25 \mu \mathrm{M}$ DCF-DA, 1 $\mathrm{mM}$ tiron, $3 \mathrm{mM}$ NAC, $2 \mathrm{nM}$ DiOC6(3), $50 \mu \mathrm{M}$ CCCP and $5 \mu \mathrm{M}$ cyclosporine A. Lysotracker, Alexa 488- or 594-conjugated secondary antibodies were from Molecular Probes (Carlsbad, CA).

\section{Preparation of DSF loaded biodegradable PEG- PLGA nanoparticles (DSFNPs)}

Single emulsification-solvent evaporation was used to encapsulate DSF into mPEG: PLGA matrix. Nanoparticles formulated in this way contain hydrophobic PLGA as a core and hydrophilic PEG on the surface.

Both disulfiram and polymer were dissolved in acetone, which serves as the dispersed phase. Aqueous phase with or without surfactant was added to the organic phase, dropwise with constant stirring. Stirring was continued to evaporate the organic phase, followed by centrifugation at $30,000 \mathrm{rpm}$ for $45 \mathrm{~min}$ to recover the nanoparticles. The pellet obtained was washed and resuspended in distilled water and the nanoparticles were then lyophilized for $48 \mathrm{~h}$. The resulting nanoparticles were stored at $4{ }^{\circ} \mathrm{C}$.

Blank nanoparticles were formulated similarly without adding the drug. HITC-iodide, a NIR dye, loaded nanoparticles were also generated in a similar manner by the addition of dye.

\section{Optimization of formulation}

The solvent evaporation technique (process), as well as the ingredients and their composition (formulation), has many variables, and we evaluated them keeping the low particle size, polydispersity index below 0.3 and high entrapment efficiency as the quality attributes. The process variables such as sonication, the speed of homogenization, evaporation time and centrifugation time, etc., were investigated first and the optimized parameters were used further. The formulation variables such as solvent, polymer molecular weight, surfactant, drug/polymer ratio and organic/aqueous phase ratio were taken into consideration and thoroughly investigated by changing one of them at a time while keeping the rest constant. The design of experiments and the effect of various variables on the particle size, PDI and entrapment efficiency are listed in the Supplementary Table 2.

\section{Measurement of particle size, size distribution, zeta potential, and morphology of mPEG-PLGA nanoparticles}

The Z-Average size and zeta potential of DSF loaded mPEG-PLGA nanoparticles (DFSNPs) suspended in water were determined using dynamic light scattering (Zetasizer 3000HS, Malvern Instruments Ltd, UK). The

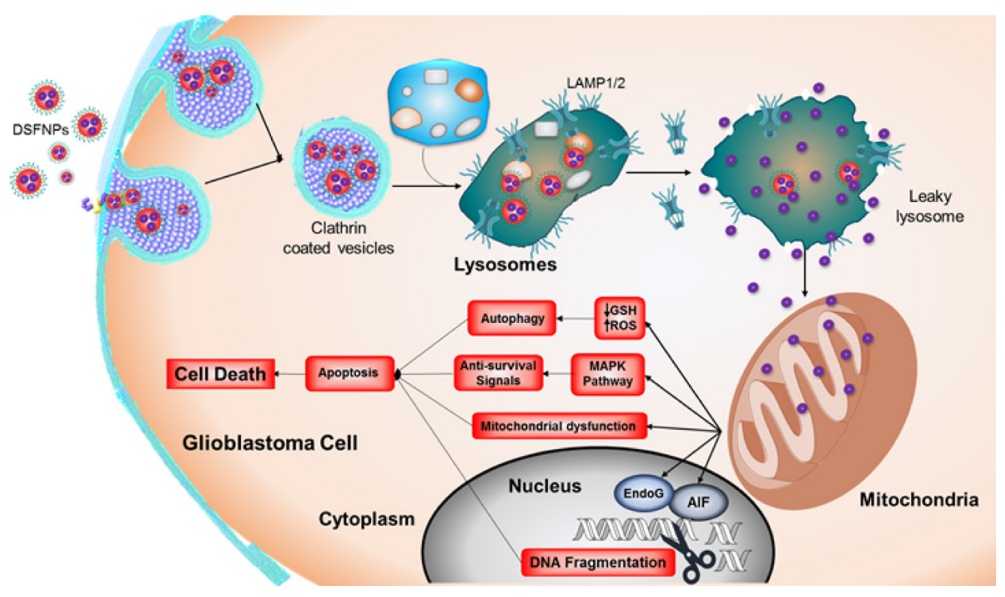

Figure 13: Schematic diagram showing the fate of DSFNPs and sequence of events leading to cytotoxicity as revealed by this study. Uptake of the DSFNPs by receptor-mediated endocytosis, their accumulation in lysosomes, lysosomal breakdown, drug entry into mitochondria, the redox events culminating in the killing of brain tumor cells are represented. 
particle size was determined using a He-Ne laser beam at a wavelength of $633 \mathrm{~nm}$ with a fixed scattering angle of $90^{\circ}$ at $25^{\circ} \mathrm{C}$. The data for a mean value for the size (z-Average) and a width parameter, Polydispersity Index (PDI) was evaluated using cumulants analysis. The PDI is a dimensionless, simple 2-parameter fit to the cumulants analysis and indicates the deviation of the measured autocorrelation function during the particle size estimation from that of monodisperse spheres having the same diameter. The PDI values range from 0 to 1 . The zeta potential values were measured by laser Doppler velocimetry at $25^{\circ} \mathrm{C}$ at the default parameters of the dielectric constant, refractive index, and viscosity of water, using a disposable capillary cell with a volume of $1 \mathrm{~mL}$. The morphology of the mPEG-PLGA nanoparticles was examined by transmission electron microscopy (Hitachi H-9500, Hitachi High Technologies America, Inc. Dallas, TX). Freeze-dried nanoparticles were dissolved in water with $0.05 \%$ Tween- 80 , and a small droplet was placed on a carbon-coated copper grid, followed by drying at room temperature before measurements were taken.

\section{Powder X-Ray diffraction}

The samples were examined at room temperature with a Rigaku Ultima III powder diffractometer. X-ray diffraction patterns were obtained by scanning a $2 \theta$ range of $5-60^{\circ}$, step size $=0.02^{\circ}$, and a scan time of $30 \mathrm{sec} /$ degree for samples PEG: PLGA- 5:45 KDa and DSF. For DSFNPs, the $2 \theta$ range and step size were kept the same with scan times increased to $60 \mathrm{sec} /$ degree. The X-ray source was $\mathrm{Cu} \mathrm{K}$ (alpha) radiation $(\lambda=1.5418 \AA)$ at an anode voltage of $40 \mathrm{kV}$ and a current of $44 \mathrm{~mA}$. The beam was then filtered by Rigaku>s Cross Beam parallel beam optics to create a monochromatic parallel beam. Data were recorded at a detector distance of $285 \mathrm{~mm}$ using a scintillation detector. The samples were prepared as standard powder mounts, and the diffractograms were processed through the software JADE v9.1.

\section{Thermal analysis}

Thermal analysis was performed to complement the data generated by X-ray diffraction, to confirm the content of DSF in the formulation and to confirm the presence of disulfiram in an amorphous state in the nanoparticle matrices. DSC is used to determine the nature and speciation of crystallinity within nanoparticles through the measurement of glass and melting point temperatures and their associated enthalpies. DSC analyses were performed using a DSC822e thermal analyzer (Mettler Toledo International Inc. Columbus, $\mathrm{OH}$ ). Samples containing approximately $5 \mathrm{mg}$ of lyophilized nanoparticles were hermetically sealed in DSC aluminum pans. DSC scans were first recorded at a heating rate of $10^{\circ} \mathrm{C} \mathrm{min}$ from $0^{\circ} \mathrm{C}$ to $100^{\circ} \mathrm{C}$ and then allowed to cool to $10^{\circ} \mathrm{C}$ to observe the exothermic phase. During thermal scans, the crucible was purged with nitrogen. The generated data were analyzed STARe software. As a control, the pure material was analyzed to observe the change of the $T_{\mathrm{m}}$ or $T_{\mathrm{g}}$.

Thermogravimetric analysis (TGA) was used to measure the physical and chemical changes of DSFNPs that occurs as a function of temperature. The procedure measures the relative change in weight of the nanoparticles with temperature changes. A Mettler TGA/SDTA851e thermobalance was used for measurements. Approximately $5 \mathrm{mg}$ of pure DSF, blank polymer or DSFNPs were heated at a flow rate of $10^{\circ} \mathrm{C} / \mathrm{min}$ in an aluminum crucible from 0 to $800^{\circ} \mathrm{C}$ under nitrogen spurge, and the mass percentages are obtained using STARe software. The mass percentage of the sample was plotted against temperature.

\section{HPLC analytical method}

HPLC based quantitative analysis was performed on Varian ProStar HPLC system equipped with a UV detector. Separation of analytes was carried on a Kinetex EVO C18 $5 \mu \mathrm{M} 100 \mathrm{~A}^{\circ}$ column $(100 \times 4.6 \mathrm{~mm}$, Phenomenex, CA) at room temperature. The mobile phase comprised of $70 \%$ acetonitrile and $30 \%$ water, which was pumped at a flow rate typically of $1 \mathrm{~mL} / \mathrm{min}$ while the injection volume was $10 \mu \mathrm{L}$. The samples were measured at $254 \mathrm{~nm}$ wavelength. The retention time for DSF and internal standard (dimethyl thiocarbamate) was at 3.6 and $2 \mathrm{~min}$ respectively.

\section{Characterization of the drug loading and entrapment efficiency of DSFNPs}

The drug loading and encapsulation efficiency of DSFNPs were determined using earlier procedures. Briefly, $1 \mathrm{mg}$ of freeze-dried DSFNPs were dissolved in $1 \mathrm{~mL}$ of methanol and incubated for $1 \mathrm{~h}$ in a $37^{\circ} \mathrm{C}$ water bath for complete extraction of the drug. The samples were centrifuged at 13,500 rpm for $5 \mathrm{~min}$, and the amount of DSF in the supernatant was determined using HPLC as described in the previous section. The drug loading and encapsulation efficiency were defined as the ratio of the amount of DSF encapsulated in nanoparticles to the total amount of DSFNPs, and the ratio of the amount of encapsulated DSF to that initially added in the process respectively. The equations are shown below.

$$
\begin{aligned}
& \% \text { LE }=\frac{\text { Drug encapsulated }}{\text { Nanoparticle weight }} * 100 \\
& \% \text { EE }=\frac{\text { Drug encapsulated }}{\text { Drug initially added }} * 100
\end{aligned}
$$

\section{In vitro release kinetics of DSFNPs}

The cumulative release of DSF from the formulation was studied with different media that mimicked the 
physiological $\mathrm{pH}$. We used simulated gastric fluid (PBS, $\mathrm{pH}$ 1.2), simulated intestinal fluid (PBS, pH 6.8) and simulated plasma (PBS, $\mathrm{pH}$ 7.4) using the dialysis method. Briefly, 10 $\mathrm{mg}$ of DSFNPs were dissolved in $2 \mathrm{~mL}$ of simulated fluid or PBS and sealed in a dialysis bag with a MW cut-off 10 $13 \mathrm{kDa}$. The dialysis bags were placed in $100 \mathrm{~mL}$ of outer medium containing $0.05 \%$ tween 80 at $37^{\circ} \mathrm{C}$. The release medium (about $0.2 \mathrm{~mL}$ ) was withdrawn at $15,30,60 \mathrm{~min}$, and 1, 2, 4, 8, 12, 24, 48, 72, 96, 120, 144, 168, and $192 \mathrm{~h}$. These samples were analyzed by HPLC.

\section{Permeability studies}

The established in vitro model of the blood brainbarrier, IMR-90, was used for determining the permeability of DSFNPs. Cells were seeded on polycarbonate 6-well Transwell ${ }^{\circledR}$ inserts with a mean pore size $3.0 \mu \mathrm{m}, 0.5 \mathrm{~mL}$ apical volume and $1.5 \mathrm{~mL}$ basolateral volume, (Corning Costar Inc., NY) at a density of $5 \times 10^{5}$ cells/well. Cells were allowed to grow at regular cell culture conditions until confluent and coherent monolayers (7-8 days) were formed; this was confirmed by measuring the transepithelial electrical resistance (TEER) of the monolayer in a growth medium at room temperature using an epithelial volt-ohm meter (EVOM, WPI Inc.). The actual TEER was calculated by subtracting the TEER value measured from a blank well without cells from the wells with cells to nullify the resistance offered by the media. The wells offering a resistance of at least 30 $\Omega . \mathrm{cm}^{2}$ were only used for further permeability studies. The studies were carried out in Hank's balanced salt solution (HBSS) buffer containing $30 \mathrm{mM}$ HEPES at $\mathrm{pH} 6.0$. Monolayers were washed with HBSS prior to the experiment, after which 0.5 and $1.2 \mathrm{~mL}$ of HBSS was placed into the upper and lower compartments respectively. At time zero, the DSFNPs were added to the apical chamber and were maintained under regular cell culture conditions. A total volume of $100 \mu \mathrm{L}$ of a solution (1 or $5 \mu \mathrm{g} / \mathrm{mL}$ ) was taken from the lower compartment at regular intervals up to $120 \mathrm{~min}$ and replaced with the same volume of fresh buffer, followed by the HPLC analysis. Apparent permeability coefficients $\left(P_{\text {app }}\right)$ were calculated by the following equation

where " $\partial \mathrm{Q} / \partial \mathrm{t}$ " is the permeability rate of the drug across the cells, " $A$ " is the diffusion area of the monolayer and " $C_{0}$ " is the initial concentration of DSF in the upper compartment.

\section{Cellular uptake of DSFNPs}

The cellular internalization was quantified through an established HPLC method. Briefly, DAOY and T98G cells were incubated in a 6-well plate until they grew to $70 \%$ confluency. Next, they were treated with 40 $\mu \mathrm{M}$ of DSF or DSFNPs for $0.5,1$, and $2 \mathrm{~h}$ or with 10 , 20 , and $40 \mu \mathrm{M}$ of DSF or DSFNPs for $1 \mathrm{~h}$. Cells were washed with cold $\mathrm{PBS}$ and kept at $-80^{\circ} \mathrm{C}$ for cell lysis.
DSF and cellular proteins were extracted by sonication followed by centrifugation. The DSF present in the supernatant was extracted into methanol followed by HPLC for quantitation. The drug amount was normalized to the protein content in each sample. Using a NIR dye encapsulated nanoparticles we performed confocal imaging as well as flow cytometric analysis to understand the extent of uptake in a time-dependent manner. Cells were incubated with HITCNPs, a NIR dye encapsulated NPs for $30 \mathrm{~min}, 1 \mathrm{~h}$ and $2 \mathrm{~h}$ followed by washing and fixing with $4 \%$ formaldehyde. Next, the cells were analyzed by flow cytometry or counterstained with DAPI followed by imaging under a multi-photon confocal microscope (Nikon Instruments Inc).

\section{In vivo real-time imaging of NIR dye- encapsulated nanoparticles}

The far-red NIR dye HITC iodide encapsulated mPEG-PLGA nanoparticles were administered to CD-1 mice at $0.2 \mu \mathrm{g}$ of HITC $/ \mathrm{kg}$ as a single $i v$ bolus injection through the tail vein. Mice were imaged at 0 and $10 \mathrm{~min}$ and $1,6,12$, and $24 \mathrm{~h}$ post- injection using a Calipers IVIS Lumina XR Imager (Caliper Life Sciences Inc., Hopkinton, MA). The fluorescence filters were set at ex. $710 \mathrm{~nm}$ and em. $800 \mathrm{~nm}$. Images were taken under auto-exposure setting with high lamp intensity after the last time point, mice were euthanized followed by cervical dislocation. All vital organs were extracted, washed twice with PBS, blotted dry and transferred to a petri dish. Ex vivo organ fluorescence intensities were recorded at similar imaging settings. Living imaging software was used to analyze the imaging data generated. Regions of interest (ROI) were drawn into the brain/skull region of $\mathrm{CD}-1$ mice, and average radiance obtained was plotted against time to get fluorescence kinetic curves of HITCNPs. Average radiance $\left(\left[\mathrm{p} / \mathrm{s} / \mathrm{cm}^{2} / \mathrm{sr}\right] /\right.$ $\left.\left[\mu \mathrm{W} / \mathrm{cm}^{2}\right]\right)$ refers to the sum of the radiance from each pixel inside the ROI divided by the number of pixels. Average radiance AUC values were calculated from the fluorescence kinetic curves and normalized to that of plain HITC dye.

$$
\text { Apparent permeability coefficient }=\frac{(\partial \mathrm{Q} / \partial \mathrm{t})}{\left(\mathrm{A}^{*} \mathrm{C}_{0}\right)}
$$

\section{Subcellular localization of DSFNPs}

Approximately $5 \times 10^{4} \mathrm{~T} 98 \mathrm{G}$ glioblastoma cells were seeded on coverslips in placed in 6-well plate and were incubated at $37^{\circ} \mathrm{C}$ for $24 \mathrm{~h}$. Then, the cells were treated for $15 \mathrm{~min}$ or $60 \mathrm{~min}$ with $100 \mathrm{mg} / \mathrm{mL}$ HITCNPs followed by the LysoTracker Red DND-99 (50 nmol/L), a marker of endolysosomal compartments or DiOC6(3) (20 nmol/L), a marker of mitochondria; the 
cells were further incubated for $30 \mathrm{~min}$. After nuclear staining with DAPI, the cells were washed, fixed with $4 \%$ paraformaldehyde, and mounted in a fluorescent mounting medium. Images were captured with a confocal microscope and were superimposed to determine the intracellular localization of the HITCNPs.

\section{Western blotting}

After trypsinization, the cell pellets were washed with cold TBS, and subjected to sonication in $50 \mathrm{mmol} / \mathrm{L}$ Tris- $\mathrm{HCl}(\mathrm{pH} 8.0)$ containing $1 \%$ glycerol, $1 \mathrm{mmol} / \mathrm{L}$ EDTA, $\beta$-mercaptoethanol, $0.5 \mathrm{mmol} / \mathrm{L}$ PMSF and 2 $\mathrm{mmol} / \mathrm{L}$ benzamidine and centrifuged. Equal protein amounts from different treatments were electrophoresed on $12 \%$ SDS-polyacrylamide gels. Proteins were electrotransferred to Immobilon-P membranes. The membranes were blocked with 5\% non-fat dry milk in Tris-buffered saline (TBS; $\mathrm{pH} 8.0$ ) containing $0.1 \%$ Tween 20 for $2 \mathrm{~h}$, and subsequently incubated with appropriate primary and secondary antibodies. The antigen-antibody complexes were visualized by enhanced chemiluminescence (Pierce Company, Woburn, MA, USA). Band intensities were quantified using the ImageJ software.

\section{Measurement of ROS}

Following treatment with DSFNPs or DMSO, T98G and DAOY cells were stained with either DHE (which is oxidized by superoxide ions into ethidium bromide and fluoresces red) or DCF-DA (which is oxidized by peroxide radicals to DCF, fluoresces green). Fluorescently labeled cells were analyzed flow cytometrically for quantification. Cells that were labeled on the coverslips were examined using fluorescence microscopy. Antimycin and hydrogen peroxide are used as positive controls for DHE and DCFDA staining respectively, whereas $\mathrm{N}$-acetyl cysteine is used as negative control in both cases.

\section{Analysis of $\Delta \Psi_{\mathrm{m}}$ dissipation}

Approximately $5 \times 10^{5}$ cells were plated in T-25 flasks and were incubated for $24 \mathrm{~h}$ followed by treatment with DSFNPs and controls. Cells were trypsinized after 24 $\mathrm{h}$ and were suspended in $1 \mathrm{~mL}$ PBS after being centrifuged and were kept on ice until staining. We followed the protocol reported by Rottenberg et al. [51] Briefly, cells were incubated with a lipophilic, cationic, fluorescent dye 3, $3^{\prime}$-Di-n-hexyloxacarbocyanine iodide $\left(\mathrm{DiOC}_{6}(3)\right)$. $\mathrm{DiOC}_{6}(2$ $\mathrm{nM})$ at $37^{\circ} \mathrm{C}$ for $20 \mathrm{~min}$, later the cells were incubated with DHE $(10 \mu \mathrm{M})$ for $10 \mathrm{~min}$. The cells were then aliquoted into two portions, $\mathrm{CCCP}(50 \mu \mathrm{M})$ was added to one portion, and were incubated for 20 more min. The cell fluorescence was measured by flow cytometry with appropriate channels. We used antimycin and $\mathrm{N}$-acetylcysteine as positive and negative controls to the drug treatment.

\section{Immunofluorescence}

Cells were seeded onto cover-slides the day before starting experiments. After treatment with DSFNPs or respective control compounds, cells were washed in PBS, prefixed in $4 \%$ paraformaldehyde for $15 \mathrm{~min}$ at $37^{\circ} \mathrm{C}$ and. Slides were then washed with PBS for three times before blocking for $1 \mathrm{~h}$ at RT with 5\% BSA and 0.03\% TritonX-100. Later, anti-LAMP1, 1:200 and anti-AIF 1:200 in PBS/1\%BSA/0.03\% Triton X-100). Slides were washed twice with PBS and incubated for $1 \mathrm{~h}$ at room temperature with the respective secondary antibodies at 1:1000 dilution. Images were taken under a confocal microscope Nikon A1MP at 100x objective magnification.

\section{Annexin V-FITC detection of apoptosis}

T98G and DAOY were harvested after treatment with DSFNPs for $24 \mathrm{~h}$, washed with PBS and resuspended in $0.5 \mathrm{~mL}$ of binding buffer (5X:10 mM HEPES pH 7.4, $150 \mathrm{mM} \mathrm{NaCl}, 2.5 \mathrm{mM} \mathrm{CaCl}_{2}, 1 \mathrm{mM} \mathrm{MgCl}_{2}, 4 \%$ BSA). The cell suspensions $(500 \mu \mathrm{l})$ were then incubated with $5 \mu \mathrm{L}$ of Annexin V-FITC (BD Bioscience) and $10 \mu \mathrm{L}$ $(50 \mu \mathrm{g} / \mathrm{mL})$ of propidium iodide for $15 \mathrm{~min}$ at $37^{\circ} \mathrm{C}$. The population of annexin $\mathrm{V}$-positive cells was evaluated by flow cytometry [52].

\section{In vitro cytotoxicity}

The DSF and DSFNPs were evaluated in parallel for assessing the cytotoxicity in six human brain cancer cell lines following a $72 \mathrm{~h}$ continuous drug exposure. The resazurin reduction assay was used to evaluate cell survival, where viable cells reduce resazurin to a highly fluorescent resorufin. The cell lines were seeded in 96well microtiter plates at a density of 4000 cells/ well and were incubated for $24 \mathrm{~h}$ before drug additions. Next, DSF and DSFNPs at ten serially diluted concentrations were added in triplicate for a further $72 \mathrm{~h}$. Next, $20 \mu \mathrm{L}$ resazurin $0.01 \%(\mathrm{w} / \mathrm{v})$ was added to each well. After $2 \mathrm{~h}$, the fluorescence was measured using a Synergy 2 multi-mode Reader (BioTek Instruments Inc.) at a 530$\mathrm{nm}$ excitation and 590-nm emission. Cell viability was expressed as a percentage of the fluorescence to that of the untreated controls, and the fluorescence of blank wells (without cells) was subtracted.

\section{Clonogenic survival assays}

A total of 400 cells/well T98G and DAOY cells were seeded in a 6-well plate, and plates were incubated for 24 $\mathrm{h}$ followed by addition of $\mathrm{IC}_{20}$ and $\mathrm{IC}_{40}$ concentrations of DSF and DSFNPs. After $24 \mathrm{~h}$, fresh media was added, and plates were incubated for 2 weeks. The cells were next washed with PBS and fixed with methanol at $-20{ }^{\circ} \mathrm{C}$ for 15 min followed by staining with $0.5 \%$ crystal violet for 20 min. Colonies were counted using the ImageJ software. 


\section{Development of orthotopic medulloblastoma xenografts and drug efficacy studies}

As the first step, the human medulloblastoma DAOY cells were engineered to express luciferase by stable transfection using the RediFect Red-FlucPuromycin lentiviral particles (Perkin Elmer) according to the manufacturer's instructions. Polybrene was used to enhance the transfection efficiency. The selection was performed in the presence of $1 \mathrm{mg} / \mathrm{ml}$ of Puromycin. Stable transfection was confirmed by luciferase activity measurements in cell lysates and imaging the cells in the presence of luciferin in IVIS.

Four-week-old male and female NCG mice, which lack T, B, and natural killer cells (triple immune-deficient) were housed in a micro ventilated caging system in a sterile environment and fed ad-libitum with standard irradiated research rodent diet and water. The animals were anesthetized using $2 \%$ isoflurane and positioned in a Benchmark (Leica) stereotactic instrument. A 27-gauge needle was then used to drill a burr hole into the skull 0.5$\mathrm{mm}$ anterior and 2-mm lateral to the bregma. DAOY-Luc2 cell suspension $\left(2 \times 10^{5}\right.$ cells in $\left.5 \mu \mathrm{PBS}\right)$ was injected in the striatum at a depth of $5 \mathrm{~mm}$ from the dural surface over 10 minutes. These mice were imaged for bioluminescence five days post tumor inoculation and were observed for stable tumor growth for the next two weeks.

The non-invasive in vivo bioluminescence measurements were performed using an IVIS-200 Caliper Imaging System. The animals lying in the prone position were given i.p. injections of D-luciferin ( $2 \mathrm{mg}$ in $100 \mu \mathrm{l}$ PBS per mouse) followed by anesthesia. Imaging was performed exactly $10 \mathrm{~min}$ after the luciferin injections. Measurements of signal intensity were obtained from a region of interest analysis encompassing the intracranial area using the Living Image software. Bioluminescence was expressed as a total radiance in photons per sec/ $/ \mathrm{cm}^{2}$ per steradian. The linearity of photon emission in our setting was optimized using a previous procedure [53].

Mice with stable and equivalent intracranial tumors were then randomly divided into four groups with 6 mice in each group, namely the vehicle controls, DSF, blank NPs, and DSFNPs. Power analysis was used to calculate that a minimum of 6 mice/group will be required to detect differences between tumor volumes assuming an $80 \%$ power, the desired $\mathrm{P}$ value of 0.05 , and a common standard deviation of 2.0. DSF or DSFNPs were given by i.p. injections at $30 \mathrm{mg} / \mathrm{kg}$ three times a week. Tumor growth of all mice was monitored once every week 10 minutes after an i.p. injections of D-luciferin as described above. Mice were monitored periodically for clinical signs of tumor burden and body weights tracked.

\section{Statistical analysis}

The data are expressed as the means \pm SEM from at least three independent experiments. Two-sided $t$-tests were used for comparisons between two groups. A value of $P<0.05$ was statistically significant at $95 \%$ confidence interval. 1way ANOVA with Dunnett multiple comparisons test was performed for in vivo tumor efficacy studies.

\section{Abbreviations}

PdI: Polydispersity Index; DLS: Dynamic light scattering; TEM: Transmission electron microscopy; SEM: Scanning electron microscopy; XRD: X-ray diffraction; DSC: Differential scanning calorimetry; TGA: Thermo gravimetric analysis; PBS: phosphate-buffered saline; FBS: fetal bovine serum; FACS: fluorescenceactivated cell sorting; HITC: the near-infrared dye 1,1'3,3,3',3'- Hexamethyl indotricarbocyanine iodide; HITCNPs: HITC encapsulated nanoparticles; DSF: Disulfiram; DSFNPs: DSF encapsulated nanoparticles; MDC: Mono dansyl cadaverine; LAMP1: Lysosomeassociated membrane glycoprotein 1; FITC: Fluorescein isothiocyanate; PE: Phycoerythrin; DCF-DA: 2', $7^{\prime}-$ Dichlorofluorescin diacetate; DHE: Dihydroethidium; CCCP: Carbonyl cyanide m-chlorophenyl hydrazine; DiOC6(3): 3,3'-Dihexyloxacarbocyanine iodide; NAC: $\mathrm{N}$-acetylcysteine; MAPK: Mitogen-activated protein kinase; AIF: Apoptosis inducing factor; CHOP: Transcription factor C/EBP homologous protein; JNK: c-Jun N-terminal kinase; ERK: Extracellular signal-regulated kinase; ACN: Acetonitrile; DCM: Dichloromethane; EA: Ethyl acetate; PVA: Polyvinylalcohol; mPEG: methoxy polyethylene glycol; PLGA: Poly lactic-co-glycolic acid; ROS: Reactive oxygen species; MGMT: O6-Methylguanine DNA methyltransferase; BBB: Blood Brain-barrier; i.p., intraperitoneal.

\section{ACKNOWLEDGMENTS AND FUNDING}

This study was supported by grants RP130266 and RP170207 from the Cancer Prevention and Research Institute of Texas (CPRIT) and funding from the Carson Leslie Research Grants for Pediatric Brain Cancers. We thank Dr. Abraham Al-Ahmad of our School for providing the IMR90 cells and helpful suggestions.

\section{CONFLICTS OF INTEREST}

The authors declare no competing financial interest.

\section{REFERENCES}

1. Mutschler J, Grosshans M, Soyka M, Rösner S. Current findings and mechanisms of action of disulfiram in the treatment of alcohol dependence. Pharmacopsychiatry. 2016; 49:137-141.

2. Zha J, Chen F, Dong H, Shi P, Yao Y, Zhang Y, Li R, Wang S, Li P, Wang W, Xu B. Disulfiram targeting lymphoid 
malignant cell lines via ROS-JNK activation as well as Nrf2 and NF-kB pathway inhibition. J Transl Med. 2014; 12:1-9.

3. Marikovsky M, Nevo N, Vadai E, Harris-Cerruti C. Cu/ $\mathrm{Zn}$ superoxide dismutase plays a role in angiogenesis. Int $\mathrm{J}$ Cancer. 2002; 97:34-41.

4. Yip NC, Fombon IS, Liu P, Brown S, Kannappan V, Armesilla AL, Xu B, Cassidy J, Darling JL, Wang W. Disulfiram modulated ROS-MAPK and NFאB pathways and targeted breast cancer cells with cancer stem cell-like properties. Br J Cancer. 2011; 104:1564-74.

5. Cvek B. Targeting Malignancies with Disulfiram (Antabuse): Multidrug Resistance, Angiogenesis, and Proteasome. Current Cancer Drug Targets. 2011; 11:332-337.

6. Liu P, Wang Z, Brown S, Kannappan V, Tawari PE, Jiang W, Irache JM, Tang JZ, Armesilla AL, Darling JL, Tang X, Wang W. Liposome encapsulated Disulfiram inhibits NFkappaB pathway and targets breast cancer stem cells in vitro and in vivo. Oncotarget. 2014; 5:7471-7485. https:// doi.org/10.18632/oncotarget.2166.

7. Li Y, Fu SY, Wang LH, Wang FY, Wang NN, Cao Q, Wang YT, Yang JY, Wu CF. Copper improves the anti-angiogenic activity of disulfiram through the EGFR/Src/VEGF pathway in gliomas. Cancer Lett. 2015; 369:86-96.

8. Yakisich JS, Siden A, Eneroth P, Cruz M. Disulfiram is a potent in vitro inhibitor of DNA topoisomerases. Biochem Biophys Res Commun. 2001; 289:586-90. https://doi. org/10.1006/bbrc.2001.6027.

9. Lin J, Haffner MC, Zhang Y, Lee BH, Brennen WN, Britton J, Kachhap SK, Shim JS, Liu JO, Nelson WG, Yegnasubramanian S, Carducci MA. Disulfiram Is a DNA Demethylating Agent and Inhibits Prostate Cancer Cell Growth. The Prostate. 2011; 71:333-343.

10. Paranjpe A, Zhang R, Ali-Osman F, Bobustuc GC, Srivenugopal KS. Disulfiram is a direct and potent inhibitor of human $\mathrm{O}(6)$-methylguanine-DNA methyltransferase (MGMT) in brain tumor cells and mouse brain and markedly increases the alkylating DNA damage. Carcinogenesis. 2014; 35:692-702.

11. Sarkaria JN, Kitange GJ, James CD, Plummer R, Calvert H, Weller M, Wick W. Mechanisms of Chemoresistance in Malignant Glioma. Clin Cancer Res. 2008; 14:2900-2908.

12. Srivenugopal KS, Rawat A, Niture SK, Paranjpe A, Velu C, Venugopal SN, Madala HR, Basak D, Punganuru SR. Posttranslational Regulation of O(6)-Methylguanine-DNA Methyltransferase (MGMT) and New Opportunities for Treatment of Brain Cancers. Mini Rev Med Chem. 2016; 16:455-464.

13. Gunasekaran S, Weinstein P, Anderson G, Parker D, Misiorowski RL, Chvapil M. Distribution of disulfiram in brain after carotid ligation in gerbils. Neuropharmacology. 1983; 22:1159-63.

14. Hothi P, Martins TJ, Chen L, Deleyrolle L, Yoon JG, Reynolds B, Foltz G. High-throughput chemical screens identify disulfiram as an inhibitor of human glioblastoma stem cells. Oncotarget. 2012; 3:1124-1136. https://doi. org/10.18632/oncotarget.707.

15. Koppaka V, Thompson DC, Chen Y, Ellermann M, Nicolaou KC, Juvonen RO, Petersen D, Deitrich RA, Hurley TD, Vasiliou V. Aldehyde dehydrogenase inhibitors: a comprehensive review of the pharmacology, mechanism of action, substrate specificity, and clinical application. Pharmacol Rev. 2012; 64:520-539.

16. ClinicalTrials.gov.Disulfiram/copper with concurrent radiation therapy and temozolomide in patients with newly diagnosed glioblastoma. https://clinicaltrials.gov/ct2/show/ NCT02715609. (10 August 2017, date last accessed).

17. ClinicalTrials.gov. Phase I study of disulfiram and copper gluconate for the treatment of refractory solid tumors involving the liver. Retrieved August 10, 2017, from https:// clinicaltrials.gov/ct2/show/NCT00742911.

18. ClinicalTrials.gov. Initial assessment of the effect of the addition of disulfiram (Antabuse) to standard chemotherapy in lung cancer. https://clinicaltrials.gov/ct2/show/NCT00 312819. (10 August 2017, date last accessed).

19. Johansson B. A review of the pharmacokinetics and pharmacodynamics of disulfiram and its metabolites. Acta Psychiatr Scand Supplementary 1992; 369:15-26.

20. Song W, Tang Z, Lei T, Wen X, Wang G, Zhang D, Deng M, Tang X, Chen X. Stable loading and delivery of disulfiram with mPEG-PLGA/PCL mixed nanoparticles for tumor therapy. Nanomedicine. 2016; 12:377-86.

21. Liu P, Brown S, Goktug T, Channathodiyil P, Kannappan V, Hugnot JP, Guichet PO, Bian X, Armesilla AL, Darling JL, Wang W. Cytotoxic effect of disulfiram/copper on human glioblastoma cell lines and ALDH-positive cancer-stem-like cells. Br J Cancer. 2012; 107:1488-1497.

22. $\mathrm{Fu} \mathrm{Y,} \mathrm{Kao} \mathrm{WJ.} \mathrm{Drug} \mathrm{release} \mathrm{kinetics} \mathrm{and} \mathrm{transport}$ mechanisms of non-degradable and degradable polymeric delivery systems. Expert Opin Drug Deliv. 2010; 7:429-444.

23. Gillich T, Acikgoz C, Isa L, Schluter AD, Spencer ND, Textor M. PEG-stabilized core-shell nanoparticles: impact of linear versus dendritic polymer shell architecture on colloidal properties and the reversibility of temperatureinduced aggregation. ACS Nano. 2013; 7:316-329.

24. Maeda $H$. The enhanced permeability and retention (EPR) effect in tumor vasculature: the key role of tumor-selective macromolecular drug targeting. Adv Enzyme Regul. 2001; 41:189-207.

25. Fasehee H, Dinarvand R, Ghavamzadeh A, EsfandyariManesh M, Moradian H, Faghihi S, Ghaffari SH. Delivery of disulfiram into breast cancer cells using folate-receptortargeted PLGA-PEG nanoparticles: in vitro and in vivo investigations. Journal of Nanobiotechnology. 2016; 14:32. https://doi.org/10.1186/s12951-016-0183-z.

26. Zhang L, Tian B, Li Y, Lei T, Meng J, Yang L, Zhang Y, Chen F, Zhang H, Xu H, Zhang Y, Tang X. A Coppermediated disulfiram-loaded $\mathrm{pH}$-triggered PEG-shedding TAT peptide-modified lipid nanocapsules for use in tumor therapy. ACS Appl Mater Interfaces. 2015; 7:25147-25161. 
27. McConville C, Tawari P, Wang W. Hot melt extruded and injection moulded disulfiram-loaded PLGA millirods for the treatment of glioblastoma multiforme via stereotactic injection. Int J Pharm. 2015; 494:73-82.

28. Costa P, Sousa Lobo JM. Modeling and comparison of dissolution profiles. Eur J Pharmaceutical Sci. 2001; 13:123-33.

29. Lippmann ES, Azarin SM, Kay JE, Nessler RA, Wilson HK, Al-Ahmad A, Palecek SP, Shusta EV. Human Blood-Brain Barrier Endothelial Cells Derived from Pluripotent Stem Cells. Nature Biotechnol. 2012; 30:783-791.

30. Yip NC, Fombon IS, Liu P, Brown S, Kannappan V, Armesilla AL, Xu B, Cassidy J, Darling JL, Wang W. Disulfiram modulated ROS-MAPK and NFkappaB pathways and targeted breast cancer cells with cancer stem cell-like properties. Br J Cancer. 2011; 104:1564-74.

31. Carter WO, Narayanan PK, Robinson JP. Intracellular hydrogen peroxide and superoxide anion detection in endothelial cells. J Leukoc Biol. 1994; 55:253-238.

32. Finkel T. Signal transduction by reactive oxygen species. J Cell Biol. 2011; 194:7-15.

33. Yuan L, Wang J, Xiao H, Wu W, Wang Y, Liu X. MAPK signaling pathways regulate mitochondrial-mediated apoptosis induced by isoorientin in human hepatoblastoma cancer cells. Food Chem Toxicol. 2013; 53:62-68.

34. Liu T, Wu L, Wang D, Wang H, Chen J, Yang C, Bao J, Wu $\mathrm{C}$. Role of reactive oxygen species-mediated MAPK and $\mathrm{NF}-\kappa \mathrm{B}$ activation in polygonatum cyrtonema lectin-induced apoptosis and autophagy in human lung adenocarcinoma A549 cells. J Biochem. 2016; 160:315-324.

35. Huang CC, Lee CC, Lin HH, Chen MC, Lin CC, Chang JY. Autophagy-regulated ROS from xanthine oxidase acts as an early effector for triggering late mitochondria-dependent apoptosis in cathepsin S-targeted tumor Cells. PLoS One. 2015; 10:e128045.

36. Webster KA. Mitochondrial membrane permeabilization and cell death during myocardial infarction: roles of calcium and reactive oxygen species. Future cardiology. 2012; 8:863-884.

37. Circu ML, Aw TY. Reactive oxygen species, cellular redox systems ans apoptosis. Free Rad Biol Med. 2010; 48:749-762.

38. Karna P, Zughaier S, Pannu V, Simmons R, Narayan S, Aneja R. Induction of reactive oxygen species-mediated autophagy by a novel microtubule-modulating agent. J Biol Chem. 2010; 285:18737-18748.

39. Waldmeier PC, Feldtrauer JJ, Qian T, Lemasters JJ. Inhibition of the mitochondrial permeability transition by the nonimmunosuppressive cyclosporin derivative NIM811. Mol Pharmacol. 2002; 62:22-29.

40. Ho S, Clipstone N, Timmermann L, Northrop J, Graef I, Fiorentino D, Nourse J, Crabtree GR. The Mechanism of Action of Cyclosporin A and FK506. Clin Immunol and Immunopathol. 1996; 80:S40-S45.

41. Zhou YY, Li Y, Jiang WQ, Zhou LF. MAPK/JNK signalling: a potential autophagy regulation pathway. Bioscience Reports. 2015; 35:e0199.
42. Zhang Y, Chen F. Reactive Oxygen Species (ROS), Troublemakers between Nuclear Factor- $\kappa \mathrm{B}(\mathrm{NF}-\kappa \mathrm{B})$ and c-Jun NH2-terminal Kinase (JNK). Cancer Res. 2004; 64:1902-1905.

43. Cheng JQ, Jiang X, Fraser M, Li M, Dan HC, Sun M, Tsang BK. Role of X-linked inhibitor of apoptosis protein in chemoresistance in ovarian cancer: possible involvement of the phosphoinositide-3 kinase/Akt pathway. Drug Resist Updat. 2002; 5:131-146.

44. Huang WC, Hung MC. Induction of Akt activity by chemotherapy confers acquired resistance. J Formos Med Assoc. 2009; 108:180-194.

45. Lee ER, Kim JY, Kang YJ, Ahn JY, Kim JH, Kim BW, Choi HY, Jeong MY, Cho SG. Interplay between PI3K/Akt and MAPK signaling pathways in DNA-damaging drug-induced apoptosis. Biochimica et Biophysica Acta - Molecular Cell Research. 2006; 1763:958-968.

46. Nobel CS, Kimland M, Nicholson DW, Orrenius S, Slater AF. Disulfiram is a potent inhibitor of proteases of the caspase family. Chem Res Toxicol. 1997; 10:1319-1324.

47. Carmody RJ, Cotter TG. Oxidative stress induces caspaseindependent retinal apoptosis in vitro. Cell Death Differ. 2000; 7:282-291.

48. Candé C, Cohen I, Daugas E, Ravagnan L, Larochette N, Zamzami N, Kroemer G. Apoptosis-inducing factor (AIF): a novel caspase-independent death effector released from mitochondria. Biochimie. 2002; 84:215-222.

49. Joza N, Susin SA, Daugas E, Stanford WL, Cho SK, Li CY, Sasaki T, Elia AJ, Cheng HY, Ravagnan L, Ferri KF, Zamzami N, Wakeham A, et al. Essential role of the mitochondrial apoptosis-inducing factor in programmed cell death. Nature. 2001; 410:549-554.

50. Shih CM, Wu JS, Ko WC, Wang LF, Wei YH, Liang HF, Chen YC, Chen CT. Mitochondria-mediated caspaseindependent apoptosis induced by cadmium in normal human lung cells. J Cell Biochem. 2003; 89:335-347.

51. Rottenberg H, Wu S. Quantitative assay by flow cytometry of the mitochondrial membrane potential in intact cells. Biochim Biophys Acta. 1998; 1404:393-404.

52. Punganuru SR, Madala HR, Venugopal SN, Samala R, Mikelis C, Srivenugopal KS. Design and synthesis of a C7-aryl piperlongumine derivative with potent antimicrotubule and mutant p53-reactivating properties. Eur J Med Chem. 2016; 107:233-44.

53. Aswendt M, Adamczak J, Couillard-Despes S, Hoehn M. Boosting bioluminescence neuroimaging: an optimized protocol for brain studies. PLoS One. 2013; 8:e55662.

54. Kuroda JI, Kuratsu JI, Yasunaga M, Yoshikatsu K, Kenmotsu H, Sugino T, Matsmura Y. Antitumor effect of NK012, a 7-ethyl-10-hydroxycamptothecin-incorporating polymeric micelle, on U87MG orthotopic glioblastoma in mice compared with irinotecan hydrochloride in combination with bevacizumab. Clin Cancer Res. 2010; 521-529. 